\title{
Embedding a Native State into a Random Heteropolymer Model: The Dynamic Approach
}

\author{
Z. Konkoli ${ }^{1,2}$ and J. Hertz ${ }^{2}$ \\ ${ }^{1}$ Department of Applied Physics, \\ Chalmers University of Technology and Göteborg University, \\ SE 41296 Göteborg, Sweden \\ ${ }^{2}$ NORDITA, Blegdamsvej 17, DK 2100 København, Denmark
}

(October 22, 2018)

\begin{abstract}
We study a random heteropolymer model with Langevin dynamics, in the supersymmetric formulation. Employing a procedure similar to one that has been used in static calculations, we construct an ensemble in which the affinity of the system for a native state is controlled by a "selection temperature" $T_{0}$. In the limit of high $T_{0}$, the model reduces to a random heteropolymer, while for $T_{0} \rightarrow 0$ the system is forced into the native state. Within the Gaussian variational approach that we employed previously for the random heteropolymer, we explore the phases of the system for high and low $T_{0}$. For high $T_{0}$, the system exhibits a (dynamical) spin glass phase, like that found for the random heteropolymer, below a temperature $T_{g}$. For low $T_{0}$, we find an ordered phase, characterized by a nonzero overlap with the native state, below a temperature $T_{n} \propto 1 / T_{0}>T_{g}$. However, the random-globule phase remains locally stable below $T_{n}$, down to the dynamical glass transition at $T_{g}$. Thus, in this model, folding is rapid for temperatures between $T_{g}$ and $T_{n}$, but below $T_{g}$ the system can get trapped in conformations uncorrelated with the native state. At a lower temperature, the ordered phase can also undergo a dynamical glass transition, splitting into substates separated by large barriers.

05.70.Ln, 87.14.Ee
\end{abstract}

\section{INTRODUCTION}

The protein folding process is relevant for all aspects of life: once read off from the RNA chain, proteins perform a variety of functions, from mechanical work to attacking viruses. [1] The key factor which determines the function of a protein molecule is its $3 \mathrm{D}$ structure, which, in turn, is determined by the sequence of amino acids forming the protein chain. [2-5] Furthermore, a protein that has been denatured (by stretching it for example) finds its native state relatively quickly. Protein folding has attracted an enormous amount of scientific attention, but still there is no generic understanding of this process. Nevertheless, one thing is clear: a proteins generally has a potential energy surface which results in a stable free energy minimum, corresponding to the native state [3].

Random heteropolymer models (RHP) have been used extensively as candidate systems which might help us to understand the generic features of the potential energy surfaces of proteins and their connection with thermodynamic [6-13] and dynamical [14-20] properties. The RHP model is characterized by quenched random monomer-monomer interactions, meant to mimic the variety of interactions between amino-acids in random sequences. It turns out that the potential energy surface of the RHP is quite similar to that of a particular class of spin glasses [21]: Its complex form, with exponentially large numbers of local minima and saddle points, con- strains the motion of the system drastically, and it cannot explore its full configuration space and reach Gibbs equilibrium. In a previous paper ( $[22]$, henceforth referred to as paper I), we demonstrated, in mean field theory, the existence of a sharp transition to a "dynamical glassy state" in which the equilibration time diverges and the dynamics exhibit aging. (The potential importance of spin glass physics to proteins was first discussed in Ref. [23]). Obviously, the random heteropolymer model does not describe a protein with a native state, but it alerts us to the need to examine possible glassiness in models for protein dynamics.

Why are real proteins not glassy? Evidently, nature has tuned amino acid sequences to avoid glassy behavior. To understand how such tuning might be done, it is worthwhile to study models which contain competition between glassiness and a tendency to form a native state, by choosing interactions which are not completely random. Several studies along the lines of this suggestion have been made in statics (using the replica treatment, see, e.g., Ref. [23]). The tendency toward a particular state can be built in by choosing sequences from a distribution correlated with the native sequence $[2,24-26]$. A dynamical treatment of similar models is highly desirable, not only to help gain insight into results obtained in replica approaches, but also because knowledge of the correct thermodynamics alone may not be sufficient: it is known that in related (mean field models) static and dynamic phase diagrams can be different. Thus (at least on 
sufficiently short time scales) only a dynamical approach can describe the measurable properties of the system. In this paper we undertake such a study.

We extend the RHP model studied in $[6,7]$ to include the existence of a native state: the original random monomer-monomer interactions are biased so as to favor the native state conformation. The problem is formulated as a Langevin model. To the best of our knowledge, there is so far neither a static nor a dynamic treatment available for a model of this sort: Static studies have been based on random monomer sequences, i.e., using only $N$ random parameters, see Refs. [2,24-26], rather than the $N(N-1) / 2$ in the RHP model.

Admittedly, the model does not describe a realistic protein (e.g., it does not give rise to secondary structure such as $\alpha$-helices or $\beta$-sheets). However, it does contain important generic features: the polymeric structure and the mixture of attractive and repulsive interactions. Together, these features lead to frustration in the structural dynamics. In our view, ours is the simplest such model that includes competition between glassy and native states. As we will see, it teaches us that one can not get rid of glassiness so easily.

As in paper I, we simplify the model further by omitting three-body interactions in the polymer. (A review describing how to include three-body terms is given in [9].) The price we have to pay for this simplification is that we have to introduce a somewhat arbitrary confining potential, which we take to have a quadratic form. We adjust its strength so that the radius of gyration $R_{g}$ of a polymer of size $N$ scales like $N^{-1 / d}$, where $d$ is the dimensionality of the system. In this way we attempt to describe a globular state. Of course, we can not describe the $\theta$-point transition in such a model, but here we are only interested in transitions between different globular states.

Our formal starting point is the Martin-Siggia-Rose generating functional for the Langevin dynamics of the model [27-30], written, for convenience and compactness, in its supersymmetric form [31]. To derive equations of motion for correlation and response functions we use a variational ansatz with a quadratic action. This approach has been used to study the problem of a manifold in a random potential, in both statics $[32,33]$ and dynamics $[34,35]$.

In paper I we showed that the RHP model exhibited broken ergodicity (formally, a spontaneous supersymmetry breaking) in a low-temperature dynamical glassy phase. In the present study, with interactions biased in favor of a native state to a controlled degree, we find, in addition, a well-folded phase, if the bias is strong enough. It can coexist with either the disordered (random-globule) state or the frozen-globule glass phase, depending on the temperature. Furthermore, we find that at low temperature the native phase can itself un- dergo a dynamical freezing into a different glassy phase. In this phase the conformation of the protein is always highly correlated with the native state, but cooperative kinetic constraints still lead to a divergent equilibration time, as for the frozen-globule state.

\section{THE MODEL}

The model is defined as follows. The Langevin dynamics is assumed to be governed by a Hamiltonian $H[x]$,

$$
\partial x(s, t) / \partial t=-\delta H[x] / \delta x(s, t)+\eta(s, t) .
$$

Here $x(s, t)$ is the position of monomer $s$ at time $t$. The monomers are numbered continuously from $s=0$ to $s=N \cdot \eta(s, t)$ is Gaussian noise

$$
\left\langle\eta(s, t) \eta\left(s^{\prime}, t^{\prime}\right)\right\rangle_{T}=2 T \delta\left(s-s^{\prime}\right) \delta\left(t-t^{\prime}\right),
$$

resulting from coupling to a heat bath at temperature $T$.

The Hamiltonian $H[x]$ contains a deterministic part $H_{0}[x, \mu]$ and a random part $H[x,\{B\}] . H_{0}[x, \mu]$ is defined as

$$
H_{0}[x, \mu]=\frac{T}{2} \int_{0}^{N} d s\left\{[\partial x(s, t) / \partial s]^{2}+\mu x(s, t)^{2}\right\} .
$$

It describes the elastic properties of the chain and a confinement potential which fixes the density of the protein. The radius of gyration $R_{g} \sim \mu^{-1 / 4}$, so, in order that the protein is compact, i.e., $R_{g} \sim N^{1 / d}$, we require $\mu \sim N^{-4 / d}$. Thus, since we are interested in very long proteins (to obtain the thermodynamic limit) we need to solve the model for $\mu$ close to zero.

The random part $H[x,\{B\}]$ describes the quenched random interactions between monomers,

$$
H[x,\{B\}]=\frac{1}{2} \int_{0}^{N} d s d s^{\prime} B_{s s^{\prime}} V\left(x(s, t)-x\left(s^{\prime}, t\right)\right) .
$$

We take $B_{s s^{\prime}}$ Gaussian, with variance $B^{2}$. The quenched average over $B_{s s^{\prime}}$ is performed as $\langle(.)\rangle_{B}=$ $\int \prod_{s>s^{\prime}} d B_{s s^{\prime}}() P.(\{B\}) . V(\Delta x)$ is a short-range potential, and, for simplicity, we take it to have a Gaussian form, as in Ref. [16],

$$
V(\Delta x)=\left(\frac{1}{2 \pi \sigma}\right)^{d / 2} \mathrm{e}^{-(\Delta x)^{2} / 2 \sigma}
$$

$d$ is the dimensionality of the system and $\sqrt{\sigma}$ the range of the potential. Large (small) $\sigma$ corresponds to a long (short) range potential. In particular, for $\sigma \rightarrow 0$, $V(\Delta x) \rightarrow \delta(\Delta x)$, and we recover the potential used in $[6,7,18]$. Here and in the following $\Delta x$ refers to a 
monomer-monomer distance: $\Delta x=x(s, t)-x\left(s^{\prime}, t\right)$ for a pair of monomers $s, s^{\prime}$.

We use reasoning similar to that employed in statics to define $P(\{B\})$ (see Refs. $[2,24-26]$ ), adapting it to the random-bond model:

$$
P(\{B\}) \propto \mathrm{e}^{-\frac{1}{T_{0}} H\left[x_{0}, B\right]-\frac{1}{2} \int d s d s^{\prime} B_{s s^{\prime}}^{2} / 2 B^{2}}
$$

$T_{0}$ is called the selection temperature, and $x_{0}(s)$ is some arbitrary native state conformation. Thus the symmetric bond distribution of the RHP model is distorted so as to give bigger weight to $B_{s s^{\prime}}$ 's which are attractive between monomers which lie close to each other in the configuration $x_{0}(s)$. Explicitly, the properly normalized $P(\{B\})$ is given by

$P(\{B\})=\left(2 \pi B^{2}\right)^{-N(N-1) / 4} \mathrm{e}^{-\beta_{0}^{2} B^{2} / 4 \int d s d s^{\prime} V\left(x_{0}(s)-x_{0}\left(s^{\prime}\right)\right)^{2}}$
$\times \mathrm{e}^{-\beta_{0} / 2 \int d s d s^{\prime} B_{s s^{\prime}} V\left(x_{0}(s)-x_{0}\left(s^{\prime}\right)\right)-1 / 2 \int d s d s^{\prime} B_{s s^{\prime}}^{2} / 2 B^{2}}$,

from which we see that the distribution of $B_{s s^{\prime}}$ is peaked around $B_{s s^{\prime}}^{\max }=-\beta_{0} B^{2} V\left(x_{0}(s)-x_{0}\left(s^{\prime}\right)\right)$. Thus, if monomers $s$ and $s^{\prime}$ are close in the native state $\left(V\left(x_{0}(s)-\right.\right.$ $\left.x_{0}\left(s^{\prime}\right)\right) \neq 0$ ), their coupling constant $B_{s s^{\prime}}$ is pulled down, as in a Go model [36,37]. For $T_{0} \rightarrow \infty$ we recover the RHP model. For $T_{0} \rightarrow 0, P(\{B\})$ picks a specific set of $B_{s s^{\prime}}$. For this set, by construction, $x_{0}(s)$ is the deepest minimum of $H[x,\{B\}]$ given in Eq. (4). This is the mechanism that embeds the native state $x_{0}(s)$.

This mechanism is somewhat arbitrary. However, the fact that the strength of embedding of the native state is controlled by the single parameter $T_{0}$ facilitates the study of transitions between random and native-like states (and, as we will show, of possible coexistence of such phases).

So far, the configuration $x_{0}(s)$ is arbitrary. Thus $x_{0}(s)$ has to be considered a quenched random function, to be averaged over just like $B_{s s^{\prime}}$ in order to obtain generic results. We will carry this average out later.

All our results are obtained in the thermodynamic limit, where the length $N$ of the heteropolymer chain goes to infinity. Also, for simplicity, we join the polymer ends to form a ring. This neglect of end effects is valid for a long chain.

\section{MAPPING TO THE FIELD THEORY}

To solve the model we map the Langevin dynamics onto a supersymmetric (SUSY) field theory. Using the standard Martin-Siggia-Rose formalism [27-30] and supersymmetric (SUSY) notation $[19,20,31,38]$, the dynamical average of any observable, for fixed $\{B\}$, can be calculated as (see, e.g., Paper I for details),

$$
\begin{aligned}
& \langle\mathcal{O}[\Phi]\rangle_{T}=\int D \Phi \mathcal{O}[\Phi] e^{-S[\Phi]}, \\
& S[\Phi]=S_{1}[\Phi]+S\left[\Phi, x_{0},\{B\}\right]
\end{aligned}
$$

where

$$
\begin{aligned}
& S_{1}[\Phi]=1 / 2 \int d s d 1 d s^{\prime} d 2 \Phi(s, 1) K_{12}^{s s^{\prime}} \Phi\left(s^{\prime} 2\right), \\
& S\left[\Phi, x_{0},\{B\}\right]=1 / 2 \int d 1 d s d s^{\prime} \times \\
& \quad \times B_{s, s^{\prime}} V\left(\Phi(s, 1)-\Phi\left(s^{\prime}, 1\right)\right),
\end{aligned}
$$

and

$$
\begin{aligned}
& K_{12}^{s s^{\prime}} \equiv \delta_{12} \delta_{s s^{\prime}} K_{1}^{s}, \quad K_{1}^{s}=T\left[\mu-(\partial / \partial s)^{2}\right]-D_{1}^{(2)}, \\
& D_{1}^{(2)}=2 T \frac{\partial^{2}}{\partial \theta_{1} \partial \bar{\theta}_{1}}+2 \theta_{1} \frac{\partial^{2}}{\partial \theta_{1} \partial t_{1}}-\frac{\partial}{\partial t_{1}} .
\end{aligned}
$$

The $\Phi(s, 1)$ denotes a superfield

$$
\begin{aligned}
\Phi(s, 1)= & x\left(s, t_{1}\right)+\bar{\theta}_{1} \eta\left(s, t_{1}\right)+ \\
& +\bar{\eta}\left(s, t_{1}\right) \theta_{1}+\bar{\theta}_{1} \theta_{1} \tilde{x}\left(s, t_{1}\right)
\end{aligned}
$$

containing the physical coordinate $x(s, t)$, the MSR auxiliary field $\tilde{x}(s, t)$, ghost fields $\eta(s, t)$ and $\bar{\eta}(s, t)$ that enforce the normalization of the distribution, and Grassmann variables $\theta$ and $\bar{\theta}$. We use the notation $1 \equiv$ $\left(\theta_{1}, \bar{\theta}_{1}, t_{1}\right)$, likewise $\int d 1 \equiv \int d \bar{\theta}_{1} d \theta_{1} d t_{1}$.

Of course, the solution can be obtained without the aid of the supersymmetric formalism, but we find it conveniently compact.

As noticed by De Dominicis [28] the expression in Eq.(8) is already normalized, so the average over the quenched disorder $B_{s, s^{\prime}}$ can be done directly on (8):

$$
\left\langle\langle\mathcal{O}[\Phi]\rangle_{T}\right\rangle_{B}=\int D \Phi \mathcal{O}[\Phi] \mathrm{e}^{-\left(S_{1}[\Phi]+S_{2}\left[\Phi, x_{0}\right]\right)}
$$

where $\exp \left(-S_{2}\left[\Phi, x_{0}\right]\right) \equiv\left\langle\exp \left(-S\left[\Phi, x_{0},\{B\}\right]\right)\right\rangle_{B}$, and

$$
\begin{aligned}
S_{2}\left[\Phi, x_{0}\right]= & -\frac{B^{2}}{4} \int d s d s^{\prime}\left[\int d 1 V\left(\Phi(s, 1)-\Phi\left(s^{\prime}, 1\right)\right)\right]^{2} \\
& -\frac{\beta_{0} B^{2}}{2} \int d s d s^{\prime} d 1 V\left(\Phi(s, 1)-\Phi\left(s^{\prime}, 1\right)\right) \times \\
& \times V\left(x_{0}(s)-x_{0}\left(s^{\prime}\right)\right) .
\end{aligned}
$$

Thus, the native state $x_{0}(s)$ enters the action in the second term of Eq. (16). Note that there is no term $\beta_{0}^{2} V\left(x_{0}(s)-x_{0}\left(s^{\prime}\right)\right)^{2}$, since it gets cancelled by a similar normalization factor for $\mathrm{P}(\{\mathrm{B}\})$ in $\mathrm{Eq}$. (7). It is useful to rewrite Eq. (16) as

$$
\begin{aligned}
S_{2}= & -\frac{B^{2}}{4} \int d^{d} x d^{d} y d 1 d 2 A_{12}^{(V)}(x, y) A_{12}^{(\delta)}(x, y) \\
& -\frac{\beta_{0} B^{2}}{2} \int d^{d} x d^{d} y d 1 A_{10}^{(V)}(x, y) A_{10}^{(\delta)}(x, y)
\end{aligned}
$$


with the notation $A_{12}^{(f)}(x, y)=\int d s f(\Phi(s, 1)-$ $x) f(\Phi(s, 2)-y), A_{10}^{(f)}(x, y)=\int d s f(\Phi(s, 1)-x) f\left(x_{0}(s)-\right.$ $y) ; f \in\{V, \delta\}$. In the long-chain limit, as discussed in Paper I (and references therein), one obtains a selfconsistent field theoretic formulation, with $S_{2}$ simplified to,

$$
\begin{aligned}
& S_{2}\left[\Phi, x_{0}\right]=\frac{B^{2}}{4} \int d^{d} x d^{d} y d 1 d 2\left[\left\langle A_{12}^{(V)}(x, y)\right\rangle\left\langle A_{12}^{(\delta)}(x, y)\right\rangle-\right. \\
& \left.-A_{12}^{(V)}(x, y)\left\langle A_{12}^{(\delta)}(x, y)\right\rangle-\left\langle A_{12}^{(V)}(x, y)\right\rangle A_{12}^{(\delta)}(x, y)\right] \\
& +\frac{\beta_{0} B^{2}}{2} \int d^{d} x d^{d} y d 1\left[\left\langle A_{10}^{(V)}(x, y)\right\rangle\left\langle A_{10}^{(\delta)}(x, y)\right\rangle\right. \\
& \left.-A_{10}^{(V)}(x, y)\left\langle A_{10}^{(\delta)}(x, y)\right\rangle-\left\langle A_{10}^{(V)}(x, y)\right\rangle A_{10}^{(\delta)}(x, y)\right] .
\end{aligned}
$$

All averages of the type $\left\langle A^{(V, \delta)}\right\rangle$ have to be calculated self-consistently with $S[\Phi]=S_{1}[\Phi]+S_{2}[\Phi]$. (We have abbreviated the double average $\left\langle\langle.\rangle_{T}\right\rangle_{B}$ simply by $\langle$.$\rangle .)$ In the limit $N \rightarrow \infty$ Eqs. (15) and (18) provide an exact description of the dynamics for an arbitrary native state $x_{0}(s)$.

\section{AVERAGE OVER NATIVE STATE CONFORMATIONS}

It is impossible to solve the model for a general native state configuration $x_{0}(s)$. We therefore consider a distribution of native states and perform the average

$$
\overline{<O\left[\Phi, x_{0}\right]>}=\int D x_{0}<O\left[\Phi, x_{0}\right]>\mathrm{e}^{-S_{0}\left[x_{0}\right]},
$$

where $S_{0}\left[x_{0}\right]$ weights each native state conformation in the ensemble as

$$
S_{0}\left[x_{0}\right]=1 / 2 \int d s x_{0}(s) K_{00}^{s s^{\prime}} x_{0}\left(s^{\prime}\right),
$$

with

$$
K_{00}^{s s^{\prime}} \equiv \delta_{s s^{\prime}}\left(\mu_{0}-\partial^{2} / \partial s^{2}\right) .
$$

The parameter $\mu_{0}$ fixes a size of the globule in this ensemble,

$$
\left\langle x_{0}(s)^{2}\right\rangle=\frac{1}{2 \sqrt{\mu_{0}}}
$$

Since the polymer ends are joined, there is translational invariance along the coordinate $s$ and $\left\langle x_{0}(s)^{2}\right\rangle$ does not depend on $s$. Thus, with this procedure, the dynamical generating functional for the problem is calculated as

$$
e^{-F_{d y n}}=\int D x_{0} D \Phi e^{-\left(S_{0}\left[x_{0}\right]+S_{1}[\Phi]+S_{2}\left[\Phi, x_{0}\right]\right)} .
$$

There is some formal similarity between the dynamical functional $F_{d y n}$ and the static replica partition function. The integration over $D x_{0}$ enters in the same way as the extra replica in the static formalism.

\section{CORRELATION FUNCTIONS}

The SUSY correlation functions

$$
\begin{aligned}
G_{12}^{s s^{\prime}} & \equiv\left\langle\Phi(s, 1) \Phi\left(s^{\prime}, 2\right)\right\rangle \\
G_{10}^{s s^{\prime}} & \equiv\left\langle\Phi(s, 1) x_{0}\left(s^{\prime}\right)\right\rangle \\
G_{00}^{s s^{\prime}} & \equiv\left\langle x_{0}(s) x_{0}\left(s^{\prime}\right)\right\rangle
\end{aligned}
$$

contain all the information we are interested in.

$G_{12}^{s s^{\prime}}$ encodes 16 correlation functions, out of which only two, correlation and response function, are independent and nonzero:

$$
\begin{aligned}
G_{12}^{s s^{\prime}}= & C\left(s, t_{1} ; s^{\prime}, t_{2}\right)+\left(\bar{\theta}_{2}-\bar{\theta}_{1}\right) \times \\
& \times\left[\theta_{2} R\left(s, t_{1} ; s^{\prime}, t_{2}\right)-\theta_{1} R\left(s^{\prime}, t_{2} ; s, t_{1}\right)\right],
\end{aligned}
$$

with

$$
\begin{aligned}
& C\left(s, t ; s^{\prime}, t^{\prime}\right) \equiv\left\langle x(s, t) x\left(s^{\prime}, t^{\prime}\right)\right\rangle, \\
& R\left(s, t ; s^{\prime}, t^{\prime}\right) \equiv\left\langle x(s, t) \tilde{x}\left(s^{\prime}, t^{\prime}\right)\right\rangle=\frac{\delta\langle x(s, t)\rangle}{\delta h\left(s^{\prime}, t^{\prime}\right)} .
\end{aligned}
$$

The field $h\left(s^{\prime}, t^{\prime}\right)$ entering the description of response function is an arbitrary external field that couples to $x\left(s^{\prime}, t^{\prime}\right)$. The fact that only two correlation functions survive is related to Ward identities originating from SUSY invariance of the original action $S$.

The supersymmetry of the theory is associated with equilibrium. One of the Ward identities resulting from SUSY is the fluctuation-dissipation theorem (FDT) which relates correlation and response functions. In the present case, the glassy state manifests itself as a spontaneous breaking of supersymmetry, leading to a modified FDT, as in previous treatments of other models $[31,34]$.

$G_{10}^{s s^{\prime}}$ describes the overlap with the native state. Due to Ward identities, only a single correlation function survives (see Appendix A for details):

$$
G_{10}^{s s^{\prime}}=\left\langle x(s, t) x_{0}\left(s^{\prime}\right)\right\rangle \equiv \phi\left(s, t_{1} ; s^{\prime}\right) .
$$

Similarly, the native state ensemble is described by

$$
G_{00}^{s s^{\prime}}=\left\langle x_{0}(s) x_{0}\left(s^{\prime}\right)\right\rangle \equiv \Gamma\left(s ; s^{\prime}\right) .
$$

$G_{12}^{s s^{\prime}}$ alone is sufficient to describe the RHP model. Here we need the two extra functions $G_{10}^{s s^{\prime}}$ and $G_{00}^{s s^{\prime}}$.

Also, in what follows, we exploit the translational invariance along the $s$ coordinate and define Fourier transforms of all correlation functions: $X\left(s, s^{\prime}\right)=$ $\int \frac{d k}{2 \pi} e^{i k\left(s-s^{\prime}\right)} X_{k}$ where $X=C, R, \phi, \Gamma$. 


\section{EQUATIONS OF MOTION}

To solve the model we proceed by making a Gaussian variational ansatz (GVA), assuming that the fields $\Phi$ are described by the approximate action

$$
\begin{aligned}
S_{v a r}= & \frac{1}{2} \int d 1 d s d 2 d s^{\prime} \Phi(s, 1)\left(G^{-1}\right)_{12}^{s s^{\prime}} \Phi\left(s^{\prime}, 2\right)+ \\
& +\int d 1 d s d s^{\prime} \Phi(s, 1)\left(G^{-1}\right)_{10}^{s s^{\prime}} x_{0}\left(s^{\prime}\right)+ \\
& +\frac{1}{2} \int d s d s^{\prime} x_{0}(s)\left(G^{-1}\right)_{00}^{s s^{\prime}} x_{0}\left(s^{\prime}\right) .
\end{aligned}
$$

Technically, this implies the following approximation for $F_{d y n}$ :

$$
F_{d y n} \approx\langle S\rangle_{v a r}+F_{v a r}
$$

where

$$
\begin{aligned}
& \mathrm{e}^{-F_{v a r}} \equiv \int D x_{0} D \Phi \mathrm{e}^{-S_{v a r}}=\mathrm{e}^{(d / 2) T r \ln G}, \\
& \langle.\rangle_{v a r}=\mathrm{e}^{F_{v a r}} \int D x_{0} D \Phi(.) \mathrm{e}^{-S_{v a r}} .
\end{aligned}
$$

The stationarity condition

$$
\frac{\delta F_{d y n}}{\delta G_{12}^{s s^{\prime}}}=0
$$

translates into the equation of motion for Green's function $G_{12}^{s s^{\prime}}$ (see Eqs. 43-46). We have derived identical equations of motion by using the approach of Ref. [35], where standard field theoretic identities (e.g. $\langle\Phi \delta S / \delta \Phi\rangle=0$ ) are used. It can be shown that for quadratic $S_{v a r}$ the two procedures give the same result. We omit this analysis here to save space.

In a corresponding equilibrium problem, the stationarity condition is also an extremum condition and provides a bound on the free energy. Here, since $F_{d y n}$ contains integrations over complex fields and Grassmann variables, the GVA does not give a bound on $F_{d y n}$. Nevertheless, it is the first step in a systematic approximation procedure, as outlined in Appendix B.

The GVA has been applied to the problem of a manifold in a random potential, in both statics $[32,33]$ and dynamics $[34,35]$. The method is exact when the dimensionality of the manifold is infinite but is only approximate for finite dimensionality. Nevertheless, even for rather low dimensionality it has been shown to be a very good approximation in the random-manifold problem, where it has been checked numerically [35]. We have shown in Paper I that the present model is closely related to the random-manifold problem. Thus, we hope that the GVA will also be reasonable here, although we have not strictly checked its validity.
Using (33), (32) and (9) gives the following expression for $F_{d y n}$ :

$$
\begin{aligned}
& F_{d y n}=\frac{d}{2} \int d s d s^{\prime} K_{00}^{s s^{\prime}} G_{00}^{s s^{\prime}}+ \\
& +\frac{d}{2} \int d s d s^{\prime} d 1 d 2 K_{12}^{s s^{\prime}} G_{12}^{s s^{\prime}}-\frac{d}{2} \operatorname{Tr} \ln G \\
& -\frac{B^{2}}{4} \int d^{d} x d^{d} y d 1 d 2\left\langle A_{12}^{(V)}(x, y)\right\rangle\left\langle A_{12}^{(\delta)}(x, y)\right\rangle \\
& -\frac{\beta_{0} B^{2}}{2} \int d^{d} x d^{d} y d 1 d 2\left\langle A_{10}^{(V)}(x, y)\right\rangle\left\langle A_{10}^{(\delta)}(x, y)\right\rangle,
\end{aligned}
$$

where all averages are to be calculated using $S_{\text {var }}$ (see Eq. 32). Performing averages, the fourth and fifth term on the right hand side of (37) become

$$
\begin{aligned}
& F_{d y n}^{(4)}=-\frac{d}{2 N} \int d 1 d 2 d s d s^{\prime} \mathcal{V}\left[\left(B_{12}^{s}+B_{12}^{s^{\prime}}\right) / 2\right], \\
& F_{d y n}^{(5)}=-\frac{\beta_{0} d}{N} \int d 1 d 2 d s d s^{\prime} \mathcal{V}\left[\left(B_{10}^{s}+B_{10}^{s^{\prime}}\right) / 2\right],
\end{aligned}
$$

where

$$
\begin{aligned}
& B_{12}^{s}=\left\langle[\Phi(s, 1)-\Phi(s, 2)]^{2}\right\rangle=G_{11}^{s s}+G_{22}^{s s}-2 G_{12}^{s s}, \\
& B_{10}^{s}=\left\langle\left[\Phi(s, 1)-x_{0}(s)\right]^{2}\right\rangle=G_{11}^{s s}+G_{00}^{s s}-2 G_{10}^{s s},
\end{aligned}
$$

and

$$
\mathcal{V}(z)=-\frac{\tilde{B}^{2}}{d}(z+\sigma)^{-d / 2}, \quad \tilde{B}^{2}=\frac{B^{2}}{2} \frac{N}{v}(4 \pi)^{-d / 2} .
$$

Performing the variational ansatz (i.e., evaluating Eq. 36) results in the following equations of motion:

$$
\begin{aligned}
& {\left[T\left(\mu+k^{2}\right)-D_{1}^{(2)}\right] G_{12}^{k}=\delta_{12}+2 \int d 3 \mathcal{V}^{\prime}\left(B_{13}\right) \times} \\
& \times\left(G_{32}^{k}-G_{12}^{k}\right)+2 \beta_{0} \mathcal{V}^{\prime}\left(B_{10}\right)\left(G_{02}^{k}-G_{12}^{k}\right), \\
& {\left[T\left(\mu+k^{2}\right)-D_{1}^{(2)}\right] G_{10}^{k}=2 \int d 2 \mathcal{V}^{\prime}\left(B_{12}\right) \times} \\
& \left(G_{20}^{k}-G_{10}^{k}\right)+2 \beta_{0} \mathcal{V}^{\prime}\left(B_{10}\right)\left(G_{00}^{k}-G_{10}^{k}\right), \\
& \left(\mu_{0}+k^{2}\right) G_{01}^{k}=2 \beta_{0} \int d 2 \mathcal{V}^{\prime}\left(B_{20}\right)\left(G_{21}^{k}-G_{01}^{k}\right), \\
& \left(\mu_{0}+k^{2}\right) G_{00}^{k}=1+2 \beta_{0} \int d 1 \mathcal{V}^{\prime}\left(B_{10}\right)\left(G_{10}^{k}-G_{00}^{k}\right),
\end{aligned}
$$

and after disentangling the SUSY notation one gets (see Paper I for related details) 


$$
\begin{aligned}
& {\left[T\left(\mu+k^{2}\right)+\partial / \partial t\right] C_{k}\left(t, t^{\prime}\right)=} \\
& 2 T R_{k}\left(t^{\prime}, t\right)+2 \int_{0}^{t} d t^{\prime \prime} \mathcal{V}^{\prime}\left[B\left(t, t^{\prime \prime}\right)\right] R_{k}\left(t^{\prime}, t^{\prime \prime}\right) \\
& +4 \int_{0}^{t} d t^{\prime \prime} \mathcal{V}^{\prime \prime}\left[B\left(t, t^{\prime \prime}\right)\right] r\left(t, t^{\prime \prime}\right)\left[C_{k}\left(t, t^{\prime}\right)-C_{k}\left(t^{\prime \prime}, t^{\prime}\right)\right] \\
& -2 \beta_{0} \mathcal{V}^{\prime}[A(t)]\left[C_{k}\left(t, t^{\prime}\right)-\phi_{k}\left(t^{\prime}\right)\right] \\
& {\left[T\left(\mu+k^{2}\right)+\partial / \partial t\right] R_{k}\left(t, t^{\prime}\right)=\delta\left(t-t^{\prime}\right)+} \\
& +4 \int_{0}^{t} d t^{\prime \prime} \mathcal{V}^{\prime \prime}\left[B\left(t, t^{\prime \prime}\right)\right] r\left(t, t^{\prime \prime}\right)\left[R_{k}\left(t, t^{\prime}\right)-R_{k}\left(t^{\prime \prime}, t^{\prime}\right)\right] \\
& -2 \beta_{0} \mathcal{V}^{\prime}[A(t)] R_{k}\left(t, t^{\prime}\right), \\
& {\left[T\left(\mu+k^{2}\right)+\partial / \partial t\right] \phi_{k}(t)=} \\
& \quad 4 \int_{0}^{t} d t^{\prime \prime} \mathcal{V}^{\prime \prime}\left[B\left(t, t^{\prime \prime}\right)\right] r\left(t, t^{\prime \prime}\right)\left[\phi_{k}(t)-\phi_{k}\left(t^{\prime \prime}\right)\right] \\
& \quad+2 \beta_{0} \mathcal{V}^{\prime}[A(t)]\left(\Gamma_{k}-\phi_{k}(t)\right) \\
& \left(\mu_{0}+k^{2}\right) \phi_{k}(t)=2 \beta_{0} \int_{0}^{t} d t^{\prime \prime} \mathcal{V}^{\prime}\left[A\left(t^{\prime \prime}\right)\right] R_{k}\left(t, t^{\prime \prime}\right) \\
& \quad\left(\mu_{0}+k^{2}\right) \Gamma_{k}=1
\end{aligned}
$$

where $B\left(t, t^{\prime}\right)$ and $A(t)$ are defined as $B\left(t, t^{\prime}\right)=\langle(x(s, t)-$ $\left.\left.x\left(s, t^{\prime}\right)\right)^{2}\right\rangle=C(s, t ; s, t)+C\left(s, t^{\prime} ; s, t^{\prime}\right)-2 C\left(s, t ; s, t^{\prime}\right)$ and $A(t)=\left\langle\left(x(s, t)-x_{0}(s)\right)^{2}\right\rangle=C(s, t ; s, t)-2 \phi(s, t ; s)+$ $\Gamma(s, s)$. Note that due to translational invariance with respect to $s$ both $B\left(t, t^{\prime}\right)$ and $A(t)$ are $s$-independent. The equations of motion for $C_{k}\left(t, t^{\prime}\right)$ and $R_{k}\left(t, t^{\prime}\right)$ are almost identical to those for the pure RHP model. Coupling to the native state enters through the terms proportional to $\beta_{0}$. Again, for large selection temperature $\beta_{0} \rightarrow 0$ and one recovers the RHP model.

\section{EXTRACTING ORDER PARAMETERS}

The equations of motion are coupled integrodifferential equations with initial conditions given by $C_{k}(0,0), \phi(0)$, and (we use Ito's convention) $R(t+\epsilon, t) \rightarrow$ 1 as $\epsilon \rightarrow 0$. To solve the equations analytically we have to consider several assumptions (which can be checked by numerical solution).

First, we make the (rather strong) standard assumptions from aging theory for spin glasses about the asymptotic behavior of the solutions: In the regime of time translational invariance (TTI),

$$
\begin{gathered}
\lim _{t \rightarrow \infty} C_{k}(t+\tau, t)=C_{k}(\tau), \\
\lim _{t \rightarrow \infty} R_{k}(t+\tau, t)=R_{k}(\tau),
\end{gathered}
$$

and, in the aging regime,

$$
\begin{aligned}
& \lim _{t \rightarrow \infty} C_{k}(t, \lambda t)=q_{k} \hat{C}_{k}(\lambda), \\
& \lim _{t \rightarrow \infty} R_{k}(t, \lambda t)=\frac{1}{t} \hat{R}_{k}(\lambda) .
\end{aligned}
$$

The validity of these assumptions could be checked numerically. Since this has been done for equations of similar type elsewhere [35], we omit it in the present analysis.

Second, it is well known that asymptotic solutions of such equations can be characterized by a few order parameters [34,35,39-41]. They are defined as

$$
\begin{aligned}
& \tilde{q}_{k}=\lim _{t \rightarrow \infty} C_{k}(t, t), \\
& q_{k}=\lim _{\tau \rightarrow \infty} C_{k}(\tau), \\
& q_{0, k}=\lim _{\lambda \rightarrow 0} q_{k} \hat{C}_{k}(\lambda), \\
& \varphi_{k}=\lim _{t \rightarrow \infty} \phi_{k}(t) .
\end{aligned}
$$

The following $k$-integrated quantities will also be useful:

$$
\begin{aligned}
\tilde{q} & \equiv \int \frac{d k}{2 \pi} \tilde{q}_{k}=\lim _{t \rightarrow \infty}\langle x(s, t) x(s, t)\rangle, \\
q & \equiv \int \frac{d k}{2 \pi} q_{k}=\lim _{\tau \rightarrow \infty} \lim _{t \rightarrow \infty}\langle x(s, t) x(s, t+\tau)\rangle, \\
q_{0} & \equiv \int \frac{d k}{2 \pi} q_{0, k}=\lim _{\lambda \rightarrow 0} \lim _{t \rightarrow \infty}\langle x(s, t) x(s, \lambda t)\rangle, \\
\varphi & \equiv \int \frac{d k}{2 \pi} \varphi_{k}=\lim _{t \rightarrow \infty}\left\langle x(s, t) x_{0}(s)\right\rangle .
\end{aligned}
$$

$\tilde{q}$ measures the size of the globule, $q$ measures the persistent correlation in the TTI regime, $q_{0}$ the asymptotic correlation in the aging regime, and $\varphi$ the overlap with native state. Also, it is useful to define

$$
\begin{aligned}
& b=2(\tilde{q}-q), \quad b_{0}=2\left(\tilde{q}-q_{0}\right), \\
& a \equiv \lim _{t \rightarrow \infty}\left\langle\left[x(s, t)-x_{0}(s)\right]^{2}\right\rangle=\tilde{q}-2 \varphi+\frac{1}{2 \sqrt{\mu_{0}}} .
\end{aligned}
$$

Third, we assume that the generalized fluctuation dissipation theorem is valid in the form

$$
\hat{R}_{k}(\lambda)=\frac{x}{T} q_{k} \frac{d \hat{C}_{k}(\lambda)}{d \lambda}
$$

$x$ could in principle depend on $k$ and $C_{k}$. However, related models have been studied in detail and they exhibit one step replica symmetry breaking with a $k$-independent $x$. This one step replica symmetry breaking ansatz in our dynamical study translates exactly to Eq. (66). 


\section{RELATING ORDER PARAMETERS}

For $t=t^{\prime}$ and $t \rightarrow \infty$ Eq. (47) gives

$$
\begin{aligned}
T(\mu & \left.+k^{2}\right) \tilde{q}_{k}=T+\frac{2}{T} \mathcal{V}^{\prime}(b)(1-x)\left(\tilde{q}_{k}-q_{k}\right) \\
& +\frac{2}{T} \mathcal{V}^{\prime}\left(b_{0}\right) x\left(\tilde{q}_{k}-q_{0, k}\right)-2 \beta_{0} \mathcal{V}^{\prime}(a)\left(\tilde{q}_{k}-\phi_{k}\right) .
\end{aligned}
$$

With $t=t^{\prime}+\tau$ and $t^{\prime} \rightarrow \infty$ and then $\tau \rightarrow \infty$ Eq. (47) becomes

$$
\begin{aligned}
T(\mu & \left.+k^{2}\right) q_{k}=\frac{2}{T}\left(\mathcal{V}^{\prime}(b)-x \mathcal{V}^{\prime}\left(b_{0}\right)\right)\left(\tilde{q}_{k}-q_{k}\right) \\
& +\frac{2}{T} \mathcal{V}^{\prime}\left(b_{0}\right) x\left(\tilde{q}_{k}-q_{0, k}\right)-2 \beta_{0} \mathcal{V}^{\prime}(a)\left(q_{k}-\phi_{k}\right)
\end{aligned}
$$

Eq. (47) in the aging regime $t^{\prime}=\lambda t$, first for $t \rightarrow \infty$ and then $\lambda \rightarrow 0$, gives

$$
\begin{aligned}
T(\mu & \left.+k^{2}\right) q_{0, k}=\frac{2}{T} \mathcal{V}^{\prime}\left(b_{0}\right)(1-x)\left(\tilde{q}_{k}-q_{k}\right) \\
& +\frac{2}{T} \mathcal{V}^{\prime}\left(b_{0}\right) x\left(\tilde{q}_{k}-q_{0, k}\right)-2 \beta_{0} \mathcal{V}^{\prime}(a)\left(q_{0, k}-\phi_{k}\right) .
\end{aligned}
$$

Eqs. (49) and (50) result in two equations for $\varphi_{k}$,

$$
\begin{aligned}
T\left(\mu+k^{2}\right) \varphi_{k}= & \frac{2}{T_{0}} \mathcal{V}^{\prime}(a)\left(\Gamma_{k}-\varphi_{k}\right) \\
\left(\mu_{0}+k^{2}\right) \varphi_{k}= & \frac{2}{T T_{0}} \mathcal{V}^{\prime}(a) x\left(q_{k}-q_{o, k}\right) \\
& +\frac{2}{T T_{0}} \mathcal{V}^{\prime}(a)\left(\tilde{q}_{k}-q_{k}\right)
\end{aligned}
$$

They are equivalent; one can chose to solve for the order parameters working with either (70) or (71). This seems a rather remarkable coincidence. We believe that it originates from the SUSY invariance of the original action $S$. For example, a similar comment holds for equations (47) and (48); they are equivalent in the TTI regime and one can derive one from the other. The 'conspiracy' of (49) and (50) not contradicting each other is very likely a similar phenomenon. Eq.(48) for $\lambda=1$ reduces to

$$
\hat{R}_{k}(1)\left(\tilde{\mu}+k^{2}+\Sigma\right)=-\left(\tilde{q}_{k}-q_{k}\right) \frac{4 \mathcal{V}^{\prime \prime}(b)}{T^{2}} \hat{r}(1),
$$

where

$$
\hat{r}(\lambda) \equiv \int \frac{d k}{2 \pi} \hat{R}_{k}(\lambda)
$$

and $\Sigma$ is defined by

$$
\Sigma=x \frac{2}{T^{2}}\left(\mathcal{V}^{\prime}(b)-\mathcal{V}^{\prime}\left(b_{0}\right)\right) .
$$

Solving these equations for the order parameters gives

$$
b=\frac{1}{\sqrt{\tilde{\mu}+\Sigma}},
$$

$$
\begin{gathered}
b_{0}=\frac{1}{x} \frac{1}{\sqrt{\tilde{\mu}}}+\frac{x-1}{x} \frac{1}{\sqrt{\tilde{\mu}+\Sigma}}, \\
\tilde{q}=\frac{b_{0}}{2}+\frac{\mathcal{V}^{\prime}\left(b_{0}\right)}{4 T^{2} \tilde{\mu}^{3 / 2}}+\frac{1}{4 \sqrt{\mu_{0}}}\left(\frac{1-\frac{\mu}{\tilde{\mu}}}{1-\frac{\mu_{0}}{\tilde{\mu}}}\right)^{2} \times \\
\times\left(2+\sqrt{\frac{\mu_{0}}{\tilde{\mu}}}\right)\left(1-\sqrt{\frac{\mu_{0}}{\tilde{\mu}}}\right)^{2}, \\
a=\frac{b_{0}}{2}+\frac{\mathcal{V}^{\prime}\left(b_{0}\right)}{4 T^{2} \tilde{\mu}^{3 / 2}}+\frac{1}{4 \sqrt{\mu_{0}}} \frac{1}{\left(1+\sqrt{\frac{\mu_{0}}{\tilde{\mu}}}\right)^{2}} \times \\
\times\left[\sqrt{\frac{\mu_{0}}{\tilde{\mu}}}\left(1+2 \sqrt{\frac{\mu_{0}}{\tilde{\mu}}}\right)+2 \frac{\mu}{\tilde{\mu}} \sqrt{\frac{\mu_{0}}{\tilde{\mu}}}\right. \\
\left.+\left(\frac{\mu}{\tilde{\mu}}\right)^{2}\left(2+\sqrt{\frac{\mu_{0}}{\tilde{\mu}}}\right)\right],
\end{gathered}
$$

$$
\tilde{\mu}=\mu+\frac{2}{T T_{0}} \mathcal{V}^{\prime}(a)
$$

and the combination of Eq. (75) and (72) gives

$$
0=\hat{r}(1)\left[T^{2}+b^{3} \mathcal{V}^{\prime \prime}(b)\right] \text {. }
$$

Furthermore, the overlap $\varphi$ with the native state is given by

$$
\varphi=\frac{1}{2 \sqrt{\mu_{0}}} \frac{1-\frac{\mu}{\tilde{\mu}}}{1+\sqrt{\frac{\mu_{0}}{\tilde{\mu}}}} .
$$

All overlap order parameters are positive. However, this result is not obvious and has to be obtained after some algebra.

These equations have two kinds of solutions. In one kind, $b=b_{0}$, so there is no glassiness (aging). For this kind of solution, the parameter $x$ is irrelevant. We call such solutions "ergodic". (While it will turn out that some of them are not truly ergodic, in the sense of describing states where the entire configuration space is visited with Boltzmann probabilities, they violate ergodicity in a rather trivial way, like a ferromagnet below the Curie temperature. We could call them "non-glassy", but we prefer not to use a negative term.)

For an ergodic solution, with $b=b_{0}, \Sigma=0$. Furthermore, $\hat{r}(\lambda)=0$, so Eqn. (80) is trivially satisfied. One then has to solve the four equations (75) and (77-79) for $b, \tilde{q}, a$ and $\tilde{\mu}$. 
The stability of such a phase against glassiness can be determined using the analysis we presented in Paper I (see Fig. 1). There, we studied a model with no nativestate bias in its interactions $\left(T_{0}=\infty\right)$ for finite $\mu$. The boundary of the glassy state as a function of $\mu$ has a form qualitatively like that in the p-spin glass as a function of field $[42,43]$. In the present model, the presence of the native state enters the calculation solely through the replacement of $\mu$ by $\tilde{\mu}$. Therefore, if a particular $T$ and $\tilde{\mu}$ fall in the glassy regime (the region below the full and dashed lines) in Fig. 1, the ergodic ansatz has to be given up.

The instability can occur in two ways, according to whether $\tilde{\mu}$ is bigger or smaller than the critical value $\tilde{\mu}_{c}$. Above $\tilde{\mu}_{c}$, the line separating glassy from ergodic regions is an Almeida-Thouless (AT) line; below it the stability condition

$$
T^{2}+b^{3} \mathcal{V}^{\prime \prime}(b)>0
$$

is violated. For $\tilde{\mu}<\tilde{\mu}_{c}$, there is no AT instability. The transition is like that for the completely random heteropolymer. To find such a transition, we have to solve for a glassy phase, characterized in part by a value of the FDT-violation parameter $x<1$ and then find where in the parameter space $x \rightarrow 1$. In the region where the $x<1$ solution exists, the associated ergodic phase is unstable and is replaced by the glassy one.

In a glassy phase, aging is present: $\hat{r}(1) \neq 0$, so the quantity in brackets in Eq. (80) has to vanish, i.e., the AT condition has to be satisfied as an equality, rather than an inequality. This so-called marginal stability condition determines $b$ as a function of temperature. In this case we have three more unknowns, $\Sigma, b_{0}$ and $x$, making a total of seven, and seven equations, (75-80), to solve for them.

We look for ergodic solutions first in the next section, and we examine their stability. Then, in the following section, we study glassy solutions (within the 1-step aging ansatz of section VII) and identify the regions in the parameter space where they hold.

\section{ERGODIC PHASES}

For ergodic phases, Eqns. (75-79) reduce to

$$
\begin{aligned}
b=b_{0}=\frac{1}{\sqrt{\tilde{\mu}}} \\
\tilde{q}=\frac{1}{2 \sqrt{\tilde{\mu}}}+\frac{\mathcal{V}^{\prime}(1 / \sqrt{\tilde{\mu}})}{4 T^{2} \tilde{\mu}^{3 / 2}}+\frac{1}{4 \sqrt{\mu_{0}}}\left(\frac{1-\frac{\mu}{\tilde{\mu}}}{1-\frac{\mu_{0}}{\tilde{\mu}}}\right)^{2} \times \\
\times\left(2+\sqrt{\frac{\mu_{0}}{\tilde{\mu}}}\right)\left(1-\sqrt{\frac{\mu_{0}}{\tilde{\mu}}}\right)^{2}
\end{aligned}
$$

$$
\begin{gathered}
a=\frac{1}{2 \sqrt{\tilde{\mu}}}+\frac{\mathcal{V}^{\prime}\left(\frac{1}{\sqrt{\tilde{\mu}}}\right)}{4 T^{2} \tilde{\mu}^{3 / 2}}+\frac{1}{4 \sqrt{\mu_{0}}} \frac{1}{\left(1+\sqrt{\frac{\mu_{0}}{\tilde{\mu}}}\right)^{2}} \times \\
\times\left[\sqrt{\frac{\mu_{0}}{\tilde{\mu}}}\left(1+2 \sqrt{\frac{\mu_{0}}{\tilde{\mu}}}\right)+2 \frac{\mu}{\tilde{\mu}} \sqrt{\frac{\mu_{0}}{\tilde{\mu}}}\right. \\
\left.+\left(\frac{\mu}{\tilde{\mu}}\right)^{2}\left(2+\sqrt{\frac{\mu_{0}}{\tilde{\mu}}}\right)\right] \\
\tilde{\mu}=\mu+\frac{2}{T T_{0}} \mathcal{V}^{\prime}(a)
\end{gathered}
$$

They can be solved numerically: given $\mu, \mu_{0}, T$ and $T_{0}$ one can find $\tilde{\mu}$, which in turn determines $\tilde{q}, b=b_{0}$ (equivalently $q=q_{0}$ ), and $\varphi$. However, it is possible to gain some analytic understanding in a few soluble limits.

In this discussion we will concentrate on the limit of small $\mu$. As we noted in paper I, if we want to confine $N$ monomers within a gyration radius $\sqrt{\tilde{q}} \propto \mu^{-1 / 4}$, we need $\mu \propto N^{-4 / d}$. Thus, for a long polymer $\mu \rightarrow 0$. We will also take $\mu=\mu_{0}$ to simplify the algebra a bit.

The pair of equations (85) and (86) fully determine $\tilde{\mu}$ as function of $T$ and $T_{0}$. For $\mu_{0}=\mu$ they take the form

$$
\begin{aligned}
a(\tilde{\mu}) & =\frac{1}{2 \sqrt{\tilde{\mu}}}+\frac{\tilde{B}^{2}}{8 T^{2} \tilde{\mu}^{3 / 2}\left(\sigma+\tilde{\mu}^{-1 / 2}\right)^{\frac{d}{2}+1}} \\
& +\frac{1}{4 \sqrt{\tilde{\mu}}}\left(1+\frac{\mu}{\tilde{\mu}}\right) \\
\tilde{\mu}(a) & =\mu+\frac{\tilde{B}^{2}}{T T_{0}(\sigma+a)^{\frac{d}{2}+1}}
\end{aligned}
$$

Given $\tilde{\mu}, T, T_{0}$ one can find the overlap with the native state $\varphi$ and the size of the polymer from (81).

\section{A. Random-globule state}

It is immediately evident that when both the temperature $T$ and the selection temperature $T_{0}$ are large, $\tilde{\mu} \approx \mu$ in (86), leading to a random-globule solution $a=b=\mu^{-1 / 2}, \tilde{q}=\mu^{-1 / 2} / 2, \varphi=0$. What is not so obvious is that in the $\mu \rightarrow 0$ limit a solution very close to this exists all the way down to very low temperatures, even for small $T_{0}$. In this subsection we examine this state in detail.

We look first for solutions of Eqs. (87) and (88) with the ansatz $\alpha \equiv \tilde{\mu} / \mu$ fixed and $\mu \rightarrow 0$. We call this the random globule ansatz, since, as will be shown, the polymer does not have any fixed conformation (it is melted), and on the average the conformations it adopts have zero overlap with the native state. (Strictly speaking, this is the only truly ergodic phase we find.) For $a$ we get,

$$
a=\frac{1}{\sqrt{\mu}}\left[\frac{3+\frac{1}{\alpha}}{4 \sqrt{\alpha}}+\mathcal{O}\left(\mu^{(d-2) / 2}\right)\right]
$$


which, after inserting into (88), gives

$$
\alpha \approx 1+\frac{\tilde{B}^{2}}{T T_{0}} \mu^{(d-2) / 4}\left(\frac{4 \sqrt{\alpha}}{3+\frac{1}{\alpha}}\right)^{d / 2+1} .
$$

Eqn. (90) can be used to calculate $\alpha$ as a function of $\mu$. One can see easily that $\alpha \rightarrow 1$ when $\mu \rightarrow 0$. This shows that our ansatz is self-consistent in the limit of small $\mu$. Also, (84) and (81) become

$$
\begin{aligned}
& \varphi=\frac{1}{2 \sqrt{\mu}}\left[\frac{\alpha-1}{2}+\mathcal{O}(\alpha-1)^{2}\right] \\
& \tilde{q}=\frac{1}{2 \sqrt{\mu}}[1+\mathcal{O}(\alpha-1)] .
\end{aligned}
$$

The normalized overlap between the polymer conformation and the native state is:

$$
\cos \theta=\frac{\lim _{t \rightarrow \infty}\left\langle x(s, t) x_{0}(s)\right\rangle}{\sqrt{\lim _{t \rightarrow \infty}\left\langle x(s, t)^{2}\right\rangle\left\langle x_{0}(s)^{2}\right\rangle}}=\frac{\varphi}{\sqrt{\tilde{q}\left(\frac{1}{2 \sqrt{\mu}}\right)}} .
$$

¿From (91) and (92) we get $\cos (\theta) \sim(\alpha-1) \sim \mu^{(d-2) / 4}$. Thus there is no overlap with native state as $\mu \rightarrow 0$.

Furthermore, to check that polymer does not freeze into some other conformation, we calculate the normalized overlap between two configurations taken at very different times,

$$
\cos \theta^{\prime}=\frac{\lim _{\tau, t \rightarrow \infty}\langle x(s, t) x(s, t+\tau)\rangle}{\sqrt{\left[\lim _{t \rightarrow \infty}\left\langle x(s, t)^{2}\right\rangle\right]^{2}}}=\frac{q}{\tilde{q}} .
$$

After rewriting

$$
q / \tilde{q}=1-\frac{b}{2 \tilde{q}}=1-\frac{1}{2 \sqrt{\tilde{\mu}}},
$$

and, using (92), we get $\cos \theta^{\prime}=\mathcal{O}(\alpha-1)$. Again, as $\mu \rightarrow 0, \cos \theta^{\prime} \rightarrow 0$. This confirms that the ansatz $\alpha=\mathcal{O}(1)$ and $\mu \rightarrow 0$ leads to a melted random-globulelike phase. This phase is identical to that found at high temperatures for the completely random heteropolymer in paper I.

The validity of the present ansatz rests upon the fact that we can solve Eq. (90). Clearly, for $\mu \rightarrow 0$ a solution can always be found, namely $\alpha=1$. Since the physically relevant $\mu$ is $\propto N^{-4 / d}$, we can always satisfy this equation, for any $T_{0}$, in the limit $N \rightarrow \infty$.

We now address briefly the question of what happens for finite $N$ (and $\mu$ ). One can easily see that Eq. (90) has two solutions when $\mu^{(d-2) / 4} /\left(T T_{0}\right)$ is not too large (e.g. by plotting the left- and right-hand side as functions of $\alpha)$. The solution close to 1 is lost when the slopes of the left- and right-hand sides become roughly equal. Evaluating these slopes leads to the condition

$$
\frac{3 \tilde{B}^{2}}{4 T T_{0}}\left(\frac{d}{2}+1\right) \mu^{\frac{d-2}{4}}<1
$$

for the existence of a random-globule-like state.

Some caution is in order. Working this out for finite $N, d=3$, and an average density of 1 , we find that the inequality (96) is violated below a temperature

$$
T_{x}=\left(\frac{\pi}{6}\right)^{1 / 2} \frac{15 \tilde{B}^{2}}{8 T_{0}} N^{-1 / 3} .
$$

With the small power of $N^{-1}$, one has to go to quite large $N$ to make this temperature very low. Thus our statement that the random-globule-like state exists for all temperatures in the $\mu \rightarrow 0$ limit may be of limited relevance for real 3-dimensional heteropolymers of the length of typical proteins. Nevertheless, here we are just considering this simple limit.

We now discuss the stability of this solution. In the large- $N$ limit, it is locally stable against spontaneous formation of a native-like state at any $T$ and $T_{0}$. However, it is unstable against glass formation at low temperatures: Since it is identical with the random-globule solution of the completely random heteropolymer problem, we can take over the result from paper I that it is unstable below a temperature $T_{g} \propto \tilde{B}$, with the constant of proportionality of order 1 . This glass temperature is independent of $T_{0}$. (In Fig. 1 this is the transition at $\tilde{\mu} \rightarrow 0$.) Thus, wherever the system is in a random-globule-like state at $T>T_{g}$, it will no longer equilibrate if the temperature is lowered below $T_{g}$. Instead, it will become glassy and its dynamics will show aging.

\section{B. Ergodic native state}

At low $T_{0}$ and $T$, one expects that the polymer should be very close to its native state, i.e., small $a$. Therefore we also look for such solutions of Eqs. (87) and (88). We will try to solve equations (87) and (88) in the limit where $\mu \rightarrow 0$ and $\tilde{\mu}$ stays finite. The limit $\mu \rightarrow 0$ turns out not to involve any subtleties when $\tilde{\mu}$ is kept constant, so we will just set $\mu=0$ from the outset. Eqs. (87) and (88) become

$$
\begin{aligned}
& a(\tilde{\mu})=\frac{3}{4 \sqrt{\tilde{\mu}}}+\frac{\tilde{B}^{2}}{8 T^{2} \tilde{\mu}^{3 / 2}\left(\sigma+\tilde{\mu}^{-1 / 2}\right)^{\frac{d}{2}+1}} \\
& \tilde{\mu}(a)=\frac{\tilde{B}^{2}}{T T_{0}(\sigma+a)^{\frac{d}{2}+1}}
\end{aligned}
$$

These equations can be solved for $\tilde{\mu}$ as function of $T$ and $T_{0}$. However, one has to keep in mind that $\mu \rightarrow 0$ has been taken. This implies that (81) and (84) become 


$$
\varphi \approx \frac{1}{2 \sqrt{\mu}}, \quad \tilde{q} \approx \frac{1}{2 \sqrt{\mu}},
$$

and, inserting (100) into (93), the normalized overlap between native state and polymer conformations, becomes $\cos \theta \approx 1$. Furthermore, because of its large overlap with the native state, the polymer is essentially frozen. This can be seen by calculating the normalized overlap between two polymer conformations after a very long time interval, as in previous section. Inserting (100) into (94) and (95) gives $\cos \theta^{\prime} \approx 1-\sqrt{\mu / \tilde{\mu}} \rightarrow 1$.

There is interesting behavior associated with the limit $\mu \rightarrow 0$ for very long polymers. When the polymer gets longer and longer $(N \rightarrow \infty)$ a finite part of the chain is not in the native state conformation, since $a$ stays constant. The rest of the chain is in the native state, which can be seen from the fact that overlap with native state approaches 1 . Thus, in the limit of a very long polymer, the fraction of chain not in the native state conformation becomes negligible: the recipe for biasing the coupling constants $B_{s s^{\prime}}$ described in chapter II works best for long polymers.

In the following we will proceed with the solution of equations (98) and (99). Before continuing, it will be useful to compactify notation a bit. Making the change of variables $\hat{X}=X / \sigma$ for $X=b, b_{0}, a, q, \tilde{q} ; \hat{Y}=Y \sigma^{2}$ for $Y=\mu, \tilde{\mu}$; and $\hat{Z}=Z \sigma^{(d-2) / 4} / \tilde{B}$ for $Z=T, T_{0}$, we get equations of the same form, with $X \rightarrow \hat{X}, Y \rightarrow \hat{Y}$ and $Z \rightarrow \hat{Z}$, but with $\sigma=1$ and $\tilde{B}=1$. Thus, without loss of generality, we can choose units with $\sigma=1$ and $\tilde{B}=1$ (and remove the hats). From now on we do this.

The working strategy for solving the equations is as follows. For fixed $T$, one can consider $T_{0}$ as a function of $\tilde{\mu}$. This can be easily done by inserting the expression for $a$ from Eq. (98) into (99), thus writing $\beta_{0}=1 / T_{0}$ as

$$
\beta_{0}(\tilde{\mu}, T)=T \tilde{\mu}[1+a(\tilde{\mu}, T)]^{d / 2+1}
$$

The four panels of Fig. 2 shows the shape of $\beta_{0}(\tilde{\mu}, T)$ as a function of $\tilde{\mu}$ for four different temperatures. We want ultimately to construct a phase diagram in the $\left(\beta_{0}, T\right)$ plane. Therefore we have to specify $T$ (one panel of the figure) and $\beta_{0}$ and ask whether one or more solutions, i.e., particular values of $\tilde{\mu}$ which solve Eq. (101), exist. For example, in panel (a) in Fig. 2, a horizontal line at $\beta_{0}>\beta_{0}^{\min }$ intersects $\beta_{0}(\tilde{\mu}, T)$ curve at two places, indicating two solutions $\tilde{\mu}=\tilde{\mu}_{1}, \tilde{\mu}_{2}$. To make the figures more readable we have shown such a horizontal line, at a particular values of $\beta_{0}$, only in panel (a). If this horizontal line is moved below $\beta_{0}^{\text {min }}$, it will never intersect the $\beta_{0}(\tilde{\mu}, T)$ curve. Thus, we can see that for every $T$, there is a value $\beta_{0}^{\min }(T)$ below which no solutions exist.

We proceed with the analysis of Fig. 2. For sufficiently high temperatures (panels (a)-(c)) there are exactly two solutions for all $\beta_{0}>\beta_{0}^{\min }$. Of these, the one with the larger value of $\tilde{\mu}$ is a stable solution (local free energy minimum) describing the ergodic native phase. For example, the solution labeled $\tilde{\mu}_{2}$ in panel (a) is of this sort. The one with the smaller value of $\tilde{\mu}$ (e.g., the one labeled by $\tilde{\mu}_{1}$ in panel (a)) is unstable. It describes a free energy maximum between the minima at the random-globule and ergodic native states. We will call such states "unstable stationary" (abbreviated US). (We have not done a static calculation to show this, but the situation here is analogous to that in an ordinary ferromagnet below the Curie temperature. There, one has three solutions of the mean field equations, one with positive, one with negative, and one with zero magnetization. The middle one, with zero magnetization, is unstable). The US state has a lower overlap with the native conformation than the ergodic-native solution does, because it has a smaller value of $\tilde{\mu}$. As $\beta_{0}$ is increased from below through $\beta_{0}^{\min }$, the native-state and US-state solutions appear together and separate. For the temperatures of panels (a)-(c), they both exist for all $\beta_{0}>\beta_{0}^{\text {min }}$.

Panel (d) (at the lowest of the temperatures) shows a more complex behavior where double-minimum structure appears. We have found numerically that this happens below $T \approx 0.20$. Here the behavior around $\beta_{0}^{\min }$ is just as in the other cases, but we note that at this temperature $\beta_{0}(\tilde{\mu}, T)$ has a second local minima at a smaller value of $\tilde{\mu}$. Thus there is a range $\beta_{1}^{\min }<\beta_{0}<\beta_{1}^{\max }$ for which there are four solutions. The rightmost one is stable and describes the ergodic-native phase, as before. Moving from right to left, the solutions alternate between stability and instability. Thus the second solution from the left represents a locally stable conformation. It is also correlated with the native state, since $\tilde{\mu}$ is finite (though we always find $\tilde{\mu} \ll 1$ in 3 dimensions). The remaining two solutions (with $\partial \beta_{0} / \partial \tilde{\mu}<0$ ) represent US states (local free energy maxima) between it and the random-globule phase in one direction and the ergodic-native phase in the other.

Plotting $\beta_{0}^{\text {min }}$ against $T$, we obtain the stability boundary indicated by the thick solid curve in Fig. 3. Within our present assumption of ergodicity, everywhere to the right of this line the ergodic-native phase is dynamically stable. One can invert the relation $\beta_{0}^{\min }(T)$, obtaining a transition temperature $T_{n}\left(\beta_{0}\right)$, the maximum temperature for which the ergodic native phase is dynamically stable. It is separated from the (also stable) randomglobule phase by a barrier, the top of which is described by the unstable solution.

In Fig. 3 we also indicate the region in the $\left(\beta_{0}, T\right)$ plane where the second locally-stable solution is found. This region has the form of a kind of sliver extending out toward large $\beta_{0}$ at low temperatures.

So far we have not examined the stability of these solutions against glassiness. As indicated above, we do this with the help of Fig. 1: Stable solutions can not lie in the range $\tilde{\mu}_{\min }<\tilde{\mu}<\tilde{\mu}_{A T}$. In Fig. 2, these limits are marked on the $\tilde{\mu}$ axes. We thus see, for example, in Panel 
(c), that the native-state solutions found for the range of $\beta_{0}$ corresponding to values of $\tilde{\mu}$ between $\tilde{\mu}_{*}$ and $\tilde{\mu}_{A T}$ are not acceptable: they violate the AT stability condition (82).

Similarly, in panel (b) the US solutions found for a range of $\beta_{0}$ values can also be seen to lie in the forbidden region. And the intermediate locally-stable states that we identified in panel (d) always lie in a glassy region.

In Fig. 3 we also plot the AT line $(80)$ in the $\left(\beta_{0}, T\right)$ plane, indicating the regions where the various kinds of ergodic solutions are forbidden. For the native-phase solutions, the forbidden region is a strip mostly at low values of $T$ (diagonally cross-hatched region between thick and AT line). However, it "wraps around" at the leftmost part of the region where those solutions are found.

The forbidden region for the US solutions occupies most of the region where these solutions occur below $T_{\max }$, the maximum temperature for a glass transition shown in Fig. 1, including the entire portion of it below $T_{g}$, the glass instability temperature of the randomglobule state.

The structure in a tiny region near the minimum value of $\beta_{0}$ for which ergodic-native solution are found is a bit complicated and cannot be seen in the top panel of Fig. 3. Therefore, the lower panel shows an enlargement of this region.

In summary, we have found four kinds of ergodic solutions. One essentially describes a random globule state. It is locally stable (in the limit of a large globule) at all $T$ and $\beta_{0}$ against condensation into a native-like state, but unstable against glass formation everywhere below a transition temperature $T_{g}$. The second kind of solution describes a phase which is highly correlated with the native state conformation, and it is stable in most of the region where the solution exists. The third kind of solution describes a locally stable state, correlated with the native state but more weakly so than the ergodic native phase just described. It is never stable against glass formation. Finally, there are unstable solutions, found whenever the ergodic-native solutions exist. They describe US states, free energy maxima between pairs of the previously-described solutions. However, in a large part of the region where these solutions are found (roughly, everywhere below $T_{\max } \approx T_{g}$ ) they violate the AT stability condition and so are not physically relevant.

Outside the regions where these ergodic solutions are allowed, we have to look for glassy solutions. We do this in the next section.

\section{GLASSY PHASES}

In a glassy phase, $\hat{r}(1) \neq 0$ and Eq. (80) has to be kept, which gives,

$$
T^{2}=-b^{3} \mathcal{V}^{\prime \prime}(b)
$$

Also, equations (74), (75) and (76) can be rewritten in the form

$$
\begin{aligned}
& \frac{\mathcal{V}^{\prime}(b)-\mathcal{V}^{\prime}\left(b_{0}\right)}{b_{0}-b}=\frac{T^{2}}{2} \frac{\sqrt{\tilde{\mu}}}{b}\left(\frac{1}{b}+\sqrt{\tilde{\mu}}\right), \\
& b_{0}-b=\frac{1}{x}\left(\frac{1}{\sqrt{\tilde{\mu}}}-b\right) .
\end{aligned}
$$

and, with $\mu_{0}=\mu,(78)$ and (79) become

$$
\begin{aligned}
& a=\frac{b_{0}}{2}+\frac{1}{8 T^{2} \tilde{\mu}^{3 / 2}\left(1+b_{0}\right)^{\frac{d}{2}+1}}+\frac{1}{4 \sqrt{\tilde{\mu}}}\left(1+\frac{\mu}{\tilde{\mu}}\right) \\
& \tilde{\mu}=\mu+\frac{1}{T T_{0}(1+a)^{\frac{d}{2}+1}}
\end{aligned}
$$

The above equations can be solved as follows. Eq. (102) gives $b$ as a function of $T$, and then (103), (105) and (106) can be used to find $b_{0}$ and $\tilde{\mu}$ as functions of $T$ and $T_{0}$. Once $b_{0}$ and $\tilde{\mu}$ are found one can calculate $\tilde{q}$ as

$$
\begin{aligned}
\tilde{q}= & \frac{b_{0}}{2}+\frac{1}{8 T^{2} \tilde{\mu}^{3 / 2}\left(1+b_{0}\right)^{\frac{d}{2}+1}}+ \\
& +\frac{1}{4 \sqrt{\mu}}\left(2+\sqrt{\frac{\mu}{\tilde{\mu}}}\right)\left(1-\sqrt{\frac{\mu}{\tilde{\mu}}}\right)
\end{aligned}
$$

As in our analysis of ergodic solutions in the preceding section, we will try two types of ansatz: one with $\tilde{\mu} / \mu=$ const as $\mu \rightarrow 0$ and one with $\tilde{\mu}=$ const as $\mu \rightarrow 0$ leading to what we call frozen-globule and glassy native phases, respectively.

\section{A. Frozen-globule phase}

The limit where $\alpha=\tilde{\mu} / \mu$ is kept constant and $\mu \rightarrow 0$ is easily treated. Eq. (102) stay the same, while Eq. (103) gives

$$
\frac{\mathcal{V}(b)-\mathcal{V}\left(b_{0}\right)}{b_{0}-b} \approx \frac{T^{2} \sqrt{\alpha}}{2 b^{2}} \sqrt{\mu}
$$

Since $b$ is kept fixed the only solution of equation above is $b_{0} \rightarrow \infty$ as

$$
b_{0} \approx \frac{\psi(b)}{\sqrt{\alpha \mu}}
$$

where $\psi(b)$ is a function which depends only on $b$, as $\mu$ is sent to 0. Inserting (109) into (106) gives

$$
\alpha=1+\mathcal{O}\left(\mu^{(d-2) / 4}\right)
$$


and $\alpha$ stays very close to 1 , as in the ergodic random globule case. Also, $\varphi$ is given by (91), while (107) gives

$$
\tilde{q}=\frac{1}{2 \sqrt{\mu}}[\psi(b)+\mathcal{O}(\alpha-1)]
$$

which can be compared with ergodic globule result, Eq. (92). Eq. (93) stays the same, and one gets $\cos \theta \sim$ $\alpha-1$ which goes to zero as $\mu \rightarrow 0$. There is no overlap with native state. Does the system freeze into some other configuration? To find out, we calculate overlap angles between configurations at time $t$ and a much later time $t^{\prime}$. As discussed in section VII there are two ways in which the limit $t, t \rightarrow \infty$ can be taken, leading to $q_{0} \neq q$.

In the first limit, the equivalent of Eq. (94) for the ansatz used here reads

$$
\cos \theta_{g}^{\prime}=\frac{\lim _{\lambda, t \rightarrow \infty}\langle x(s, t) x(s, \lambda t)\rangle}{\sqrt{\left[\lim _{t \rightarrow \infty}\left\langle x(s, t)^{2}\right\rangle\right]^{2}}}=\frac{q_{0}}{\tilde{q}}
$$

Using Eq. (64), we can write $\cos \theta_{g}^{\prime}=1-b_{0} / 2 \tilde{q}$, and Eqs (111) and (109) give $\cos \theta_{g}^{\prime} \sim \alpha-1$ which goes to 0 as $\mu \rightarrow 0$. (This behavior is analogous to that found in p-spin glasses.) However, at not-too-long time scales (shorter than the waiting time), as in equation (113), the polymer is frozen:

$$
\cos \theta_{g}^{\prime \prime}=\frac{\lim _{\tau, t \rightarrow \infty}\langle x(s, t) x(s, t+\tau)\rangle}{\sqrt{\left[\lim _{t \rightarrow \infty}\left\langle x(s, t)^{2}\right\rangle\right]^{2}}}=\frac{q}{\tilde{q}}
$$

Using Eq. (64), we can write $\cos \theta_{g}^{\prime \prime}=1-b / 2 \tilde{q}$, and Eq. (111) gives $\cos \theta_{g}^{\prime \prime} \sim 1-\sqrt{\mu} b / \psi(b)$, which goes to 1 as $\mu \rightarrow 0$. Thus, this glassy phase has no overlap with the native state.

As discussed above, there is an upper temperature limit $T_{g}$ (independent of $\beta_{0}$ ) above which this phase melts, leaving the system in the random-globule state. $T_{g}$ can be found from Eqs. (102) and (103), using $b_{0} \rightarrow \infty$ and (104) with $x \rightarrow 1$. This leads to a value $b=2 /\left(\frac{1}{2} d-1\right)=\mathcal{O}(1)$ at the transition and $T_{g}=2\left(\frac{1}{2} d-1\right)^{\frac{1}{2}\left(\frac{1}{2} d-1\right)} /\left(\frac{1}{2} d+1\right)^{\frac{1}{2}\left(\frac{1}{2} d+1\right)}$. For $d=3$, $T_{g} \approx 0.535$.

\section{B. Glassy native states}

We also have to study the possible glassy phases with overlap with the native state, i.e., with finite $\tilde{\mu}$ (and, accordingly, finite $a$ ) when $\mu \rightarrow 0$. In such a phase, as in the ergodic native-like states described above, the system moves only in the neighborhood of the native state configuration. However, in a "glassy native" state even this restricted motion is strongly suppressed by the complexity of the local potential energy surface, and a glassy phase results.
As in the ergodic ansatz, the limit $\mu \rightarrow 0$ introduces no problems. Eqns. (102) and (103) remain the same as in the frozen-globule case, while the equations for $a$ and $\tilde{\mu},(105)$ and (106) become

$$
\begin{aligned}
& a(\tilde{\mu})=\frac{b_{0}}{2}+\frac{1}{8 T^{2} \tilde{\mu}^{3 / 2}\left(1+b_{0}\right)^{\frac{d}{2}+1}}+\frac{1}{4 \sqrt{\tilde{\mu}}} \\
& \tilde{\mu}(a)=\frac{1}{T T_{0}(1+a)^{\frac{d}{2}+1}}
\end{aligned}
$$

Again, Eq. (102) specifies $b$ as a function of $T$, and (103), (114) and (115) determine $b_{0}$ and $\tilde{\mu}$ as functions of $T$ and $T_{0}$. $\tilde{q}$ and $\varphi$ are given by $\varphi, \tilde{q} \approx 1 /(2 \sqrt{\mu})$.

The overlap with the native state is the largest possible: $\cos \theta_{g}=1$, as can be easily seen from Eq. (93) and the values for $\varphi$ and $\tilde{q}$ we have just given. The overlap between two conformations at very different times also takes its largest possible value. From Eqs. (112) and (113), knowing that $b_{0}$ and $b$ do not depend on $\mu$ we have $\cos \theta_{g}^{\prime}=1-b_{0} / 2 \tilde{q} \approx 1-b_{0} \sqrt{\mu} \rightarrow 1$ and $\cos \theta_{g}^{\prime \prime}=1-b / 2 \tilde{q} \approx 1-b \sqrt{\mu} \rightarrow 1$. Thus, the polymer is frozen almost everywhere into the native conformation. However, the freezing is not total, since $a$ in (114) is not zero. Furthermore, there is aging in the system, since $x$ in Eq. (104) is not equal to 1.

We turn now to the solution of the equations (102), (103), (114) and (115). As for the corresponding ergodic phases we have to resort to numerical solution; here we describe the analysis. The working strategy is similar to the one presented in subsection IX.B; the goal is to find $\beta_{0}$ as function of $\tilde{\mu}$ for fixed $T$ since, as in the ergodic native case, extrema of the function $\beta_{0}(\tilde{\mu}, T)$ govern the phase boundaries.

The procedure for finding value of the function $T_{0}(\tilde{\mu}, T)$ is as follows. Eq (102) determines $b$ as a function of $T$, to be referred to as $b(T)$. Once $b(T)$ is found from (102) it is inserted into Eq. (103), which determines $b_{0}(T, \tilde{\mu})$. The value found for $b_{0}$ is inserted into Eq. (114) to find $a$, and finally $\beta_{0}=1 / T_{0}$ is calculated from Eq. (115). Thus, at each temperature for which glassy solutions are possible, we can construct a graph of $\beta_{0}(\tilde{\mu})$, as we did for ergodic solutions in Fig. 2. We have used Mathematica to do these calculations. We can use these curves, together with the ergodic ones previously analyzed, to identify the possible states of the system at a given temperature and $\beta_{0}$ (Fig. 4). The procedure is fairly simple. At any given $\tilde{\mu}$, only one of the solutions is physical: In the region $\tilde{\mu}_{\text {min }}<\tilde{\mu}<\tilde{\mu}_{A T}$ one has to follow the glassy $\beta_{0}(\tilde{\mu})$ curve, while outside it one follows the ergodic one. In Fig. 4 the physical solution is indicated as the thick dashed curve. One then looks for solutions as intersections of this curve with a horizontal line at a given value of $\beta_{0}$, as done previously (e.g., as in Fig. 2, panel (a)) within the ergodic ansatz.

In Fig. 4 this procedure is shown for several different values of $T$. In the first panel, $T$ lies just a little below 
$T_{\max }$ (as in Panel (b) of Fig. 2). Suppose we start in the ergodic native phase at large $\beta_{0}$ and then lower $\beta_{0}$. (In Fig. 5, this would correspond to moving along a horizontal line (constant $T$ ) slightly above point $\mathrm{B}$ in panel (b) or (c)). We can lower $\beta_{0}$ all the way down to $\beta_{0}^{\text {min }}$ without encountering an AT instability. So, just as in the ergodic analysis of section X.B, beyond $\beta_{0}^{\text {min }}$ the ergodic native phase melts into the random-globule phase.

In the same panel we can also analyze the what happens to the unstable stationary state in the same range of $\beta_{0}$ for this temperature. At very large $\beta_{0}$ we have an ergodic solution, but as we lower $\beta_{0}$ we pass through a range of $\tilde{\mu}$, between $\tilde{\mu}_{\text {min }}$ and $\tilde{\mu}_{A T}$, where the ergodic solution is unstable against glassiness. In this region we must follow the glassy curve instead of the ergodic one. We interpret this glassy solution in the following way: The free energy landscape near the US maximum becomes rough in this range of values of $\beta_{0}$ (at this temperature), the same way the free energy landscape near the minimum corresponding to a thermodynamic phase becomes rough in a glassy state. We call it a "glassy US state".

The next panel is for a slightly lower temperature (but still above $T_{g}$ ). Here, as we lower $\beta_{0}$ in the ergodicnative phase, we reach an AT instability before we get all the way down to the minimum on the ergodic curve. (In Fig. 5, this would correspond to moving on a line of constant $T$, meeting the AT line somewhere between points A and B in panel (b) or (c)). Furthermore, the only available glassy solution for $\tilde{\mu}<\tilde{\mu}_{A T}$ is one with negative $\partial \beta_{0} / \partial \tilde{\mu}$, that is, it corresponds to the kind of glassy US state discussed above. As this is not a stable phase, we conclude that for this $T$, the minimum value of $\beta_{0}$ lies at this AT line, and beyond it there is no stable native-like state. We can follow the glassy US state back up to larger $\beta_{0}$, seeing that we eventually cross over to a normal (non-glassy) transition state.

In the last panel, the temperature is lowered a bit more (below $T_{g}$ ). Again, starting in the ergodic native phase at large $\beta_{0}$ and lowering $\beta_{0}$, we encounter an AT instability and a glassy solution appears. (Equivalently, in Fig. 5 one moves on a horizontal line somewhere below $T_{g}$ until meeting the AT line for the first time.) For smaller $\beta_{0}$, we switch to the glassy curve, which has positive $\partial \beta_{0} / \partial \tilde{\mu}$, describing a glassy native phase. We can follow this curve down to its minimum $\beta_{0}$, beyond which no phases correlated with the native phase exist. But, of course, following it back up toward large $\beta_{0}$ on the unstable branch, we can identify the glassy US state between the phase correlated with the native state and the one uncorrelated with it. (Above $T_{g}$, the latter is the random-globule phase; below it, it is the frozen-globule phase.)

\section{FEATURES OF THE PHASE DIAGRAM}

The phase structure implied by this simple model is not so simple. Fig. 5 shows the phase diagram constructed from the above analysis. For clarity, we show in the top panel only the solutions that correspond to stable phases. The second panel shows the details in the region where the ergodic-native, glassy-native, randomglobule and frozen globule states come together (or nearly so). The third panel shows the regions where ergodic and glassy US states are found.

There are six distinct regions in the phase diagram. In region I (high $T$, small $\beta_{0}$ ), the only stable phase is the random globule. In region II (small $\beta_{0}, T<T_{g}$ ) it undergoes a glass transition to the frozen-globule phase. The properties of the system in this part of the phase diagram are the same as in the completely random heteropolymer model of paper I; the bias of the interactions toward a native state does not have any effect until a (temperaturedependent) threshold $\beta_{0}^{c}(T)$ is reached. This threshold is marked on the diagram by the lines separating region I from regions III and V and region II from region VI.

To help thinking about these phases, we offer the schematic free energy-surface pictures of Fig. 6. They show how we imagine the free energy varies as a function of the native-state overlap coordinate $\varphi$. Fig. $6 \mathrm{~A}$ depicts this cross-section through the free-energy surface in region I, where there is a single smooth minimum around $\varphi=0$, representing the random-globule phase. Fig. 6B shows what happens in region II, where this phase is replaced by the frozen-globule phase. We represent this by drawing the free energy surface with many local minima. Fig. 6C shows what happens in the middle of region III, where there are two (smooth) minima, the new one corresponding to the ergodic native phase. (It will lie above or below that at $\varphi=0$ according to whether we are above or below a first-order transition that we expect to occur at a temperature $T_{1}<T_{n}$, see below.) In Fig. $6 \mathrm{D}$ we depict the situation in region IV, where both the $\varphi \approx 0$ region and the region around the maximum become rough. Fig. $6 \mathrm{E}$ represents region $\mathrm{V}$, with the native valley and the maximum rough, but the region near $\varphi=0$ still smooth. In Fig. $6 \mathrm{~F}$ (region VI), that, too, becomes rough.

An important feature is the fact that the randomglobule and frozen-globule phases remain (dynamically) stable in their respective temperature ranges for all $\beta_{0}$. Thus the horizontal line separating region I from region II continues across the diagram, separating region $\mathrm{V}$ from region VI and region II from region IV.

In each of regions II, IV, V and VI (Fig. 6, panels (b), (d), (e), (f)) there are two stable states. Thus, regions I and III are separated by the line $T_{n}\left(\beta_{0}\right)$, below or to the right of which, in addition to the random-globule state, an ergodic native state exists and is stable. In region 
IV, the frozen-globule and this ergodic native state are both stable. In region $\mathrm{V}$, the random globule state and a glassy native state are stable, while in region VI the stable states are the frozen globule and the glassy native phase.

The diagram shows some interesting fine structure in the neighborhood of the region where regions I, III, and V meet (second panel, point A). In particular, below point $\mathrm{A}$, the boundary between regions III and V (i.e., between the ergodic-native and glassy-native state) is an AT line. It comes about as can be seen in the last panel of Fig. 4, where, following the ergodic phase down from high $\beta_{0}$ and $\tilde{\mu}$, we reach $\tilde{\mu}_{A T}$ and thereafter have to switch to the glassy solution.

On the other hand, the boundary $T_{n}\left(\beta_{0}\right)$ above point $\mathrm{B}$ is reached as in the first panel of Fig. 4: one can come all the way down to the minimum value of $\beta_{0}$ for the ergodic solution before reaching $\tilde{\mu}_{A T}$. The full line continuing upwards and to the right of point $\mathrm{B}$ is an AT line which goes over into an $x=1$ line at its maximum, $T_{\max }$. Below this line US solutions become glassy.

Between points A and B, the boundary is marked by reaching $\tilde{\mu}_{A T}$ in the way shown in the second panel of Fig. 4: There is no stable ergodic native solution to the left of this line, since, upon lowering $\beta_{0}$ below $\beta_{0}^{\min }$ in Panel (b), the solution with $\partial \beta_{0} / \partial \tilde{\mu}>0$ is lost. Thus in this region the line $\mathrm{AB}$ is an $\mathrm{AT}$ line for both the native phase and the US solutions. The dashed line marks the boundary found if one ignores the AT line (i.e., it is a portion of the boundary found using the ergodic ansatz and shown in Fig. 3).

Everywhere below the AT line (both the portion AB and its extension upward to the right) is the region where the US solutions are glassy, as shown in the last panel of Fig. 5. At a given $\beta_{0}$, these features all have an onset at a temperature between $T_{g}$ and $T_{\max }$. As can be seen in Fig. 1, this is a very small temperature range. This is also the reason why there is fine structure, as shown in both the second and the third panels of Fig. 5, in such a small temperature range in the phase diagrams.

As remarked above, we have not done an equilibrium calculation, but we expect that the first-order transition temperature $T_{1}\left(\beta_{0}\right)$ where the free energies of the random-globule and ergodic-native phases are equal will also rise with $\beta_{0}$. For large $\beta_{0}$, we expect $T_{1}$, like $T_{n}$, to be proportional to $\beta_{0}$ (but $T_{1}<T_{n}$, of course).

Thus, at fairly large $\beta_{0}$, we expect the following sequence of stable states as we lower $T$ from a high value. Initially, only the random-globule state is stable. Then, below $T_{n}$, the ergodic native state is also stable, and below $T_{1}$ it becomes the lowest-free energy state. Going further down in $T$, we cross the boundary (last panel in Fig. 5) where the US state between the ergodic native and random-globule states becomes glassy (i.e., acquires a rough local free energy landscape). Very soon there- after, we cross $T_{g}$, where the random-globule state undergoes glassy freezing. Continuing, we reach a temperature where the ergodic native state undergoes glassy freezing. Finally, we reach the stability limit of this glassy-native phase, leaving the system with nowhere to go but the frozen-globule state.

What lessons are there in these findings for protein folding? We start from the assumption that the initial state in the folding process is uncorrelated with the native state (i.e., in Fig. 6 we start in a local minimum at $\varphi \approx 0$ ). Folding requires the system to find its way to the (ergodic) native state.

One feature that is evident is that such a path in configuration space always requires an uphill free-energy step. This is because either the random-globule or frozenglobule phases is always locally stable.

If we stick to our mean-field dynamical picture, where barriers are infinite, folding is, strictly speaking, impossible. In dynamical terms the "infinite" barriers translate into the fact that the equations which govern the motion of order parameters, $\varphi$ included, have basins of attraction corresponding to the plots shown in Fig. 6. For example, starting from $\varphi$ somewhere close to 0 and given a free energy profile like that in Fig. 6C, dynamical equations will never carry $\varphi$ to the large value describing the native state. On the contrary, $\varphi$ will approach 0 as time goes on.

But, if we relax this assumption and imagine finite barriers (associated with local nucleation of a native phase [44-46]), we may ask (informally) when activated motion over the barrier to nucleation is least hindered. We argue that the free energy landscape features present globally in our mean-field picture will also be relevant locally: when our calculation here finds a glassy US state, we expect that free energy surface near the true transition state will also be rough. Thus, from the preceding description of the phases and the transition states between them, we can see that folding should be easiest for large $\beta_{0}$ in a window between $T_{1}\left(\beta_{0}\right)$ and the upper boundary of the region where the US states become glassy (and passage across the transition region is kinetically impeded by the tortuous nature of the local free energy landscape). The latter boundary lies, in turn, just barely above $T_{g}$, where the landscape in the (large) portion of the configuration space uncorrelated with the native state also becomes rough, further impeding escape from it. At still lower temperatures, things become even worse, first with the onset of glassiness in the native-like region of configuration space itself and finally with the disappearance of native-like solutions. But these features probably have minor consequences, since folding will already have been so strongly impeded by the effects (with onset near $T_{g}$ ) that tend to confine it in a region of configuration space uncorrelated with the native state. 


\section{DISCUSSION}

We have introduced what we might call a generic model for a protein, based on what seems to us to be the simplest way to incorporate a tendency to form a native state in an otherwise random heteropolymer model. To make it possible to calculate typical properties, we follow previous authors $[2,24-26]$ and do not specify a particular native state, but rather an ensemble of them, constrained only by chain-entropic constraints and confinement to the appropriate volume. This ensemble is characterized by the selection temperature $T_{0}$. Our model differs from previous ones in that they are based on random-sequence heteropolymers, while we start from a model $[6,7]$ in which each monomer-monomer interaction is an independent random variable.

While it might be argued that random-sequence models are more relevant to proteins, they approach the model we consider here in the limit where the number of monomer types becomes large. Thus, what we find out about our model may be relevant to proteins (with 20 different amino acids). Of course, it is also important to study what happens away from the large-monomer-type limit; our analysis here can help in solving that more difficult problem.

Furthermore, naively, one might assume that by adjusting $N(N-1) / 2$ parameters one could imprint a native state more strongly than for models with only $N$ parameters. Our model shows that this is not necessarily true. Parts of the phase diagram are glassy, even for very low selection temperature $T_{0}$, when the native state should be strongly imprinted into the model.

Instead of the quadratic confinement term $\mu x(s, t)^{2}$ one could add three-body terms, which are commonly used to fix the globule density. It would be interesting to extend the analysis presented here to such models. Also, in our treatment, translational invariance within the globule is put in by hand. Keeping three-body terms would lead to automatic translational invariance. We have seen that if translational averaging is omitted (see paper I) then the equations become coupled in the $k$ variable and are thus a lot harder to solve.

Within out model, we have made just two approximations: the Gaussian variational ansatz of section VI, and the assumption of 1-step ergodicity breaking (analogous to 1-step replica symmetry breaking in the replica approach). Otherwise the solution is complete and exact to the accuracy we were able to achieve numerically.

Our most important result is the existence of the various different phases at large $\tilde{B} / T_{0}$, where the interactions that favor a native state are strong. While it is natural to anticipate that the native-like configurations will be thermally disrupted above a temperature of order $\tilde{B}^{2} / T_{0}$, it is not so obvious that at low temperatures there will be other impediments to efficient folding. We identify two of these:

(1) The frozen-globule state, which is uncorrelated with the native state, always exists below $T_{g}$, no matter how big $\beta_{0}$ is. This means that in a large part of configuration space, the system may be trapped in a rough energy landscape and never (in MFT) get to the nativestate region where it can fold rapidly. Furthermore, in almost the same temperature, range, we expect that the energy landscape is also rough around the transition region on the way to the correctly-folded state, further impeding the folding process. Thus, while lower temperature favors well-folded over random-globule-like configurations energetically, the rough energy landscape of the glassy phase will hinder correct folding. Our conclusion here is consistent with that of Goldstein et al. [47], who found (albeit in a different kind of model) that a large $T_{n} / T_{g}$ (or $T_{1} / T_{g}$ ) ratio favors folding.

(2) At even lower temperatures, the native state itself is unstable against a glass transition where it splits into a large number of substates. Transitions between these substates are blocked by high barriers (infinite, in MFT). A phase of this kind was found earlier by Bryngelson and Wolynes in a phenomenological model [48]. It is tempting to associate the substates with the glassy conformations observed at temperatures below $200 \mathrm{~K}$ in myoglobin [49].

Of course, MFT is an approximation. The escape from the tortuous part of the energy landscape to the smooth region will not take forever, nor will transitions between low- $T$ substates. Nevertheless, MFT does indicate when we can expect relaxational dynamics, including folding, to be slow or fast, as well as give us some insight into the physics behind these differences.

Our analysis here is a purely dynamical one. We do not compute equilibrium partition functions. A complete analysis would include such calculations, but we defer them to future work. Nevertheless, the purely dynamical analysis can reveal important properties of the system that cannot be seen in an equilibrium analysis. For example, it has been know for a long time that for a large class of models - namely, those which have a glass transition where $x \rightarrow 1$ - the dynamic and static glass transition temperatures are different $[39,42,43]$. This is expected to be the case for the transition at $T_{g}$ in our model: The equilibrium glass transition temperature is lower than the dynamical one. Thus, in a temperature range just below the dynamical $T_{g}$, the equilibrium analysis does not reveal the slow dynamics (accompanied by aging) that we are able to identify and analyze here.

In other glassy models for which it has been possible to do a more complete analysis $[34,35,39,42,43]$, the static and dynamic transitions coincide when they occur as a result of an Almeida-Thouless instability (the marginal stability condition, Eqn 80) [50]. This is the case here at the phase boundary where the native-like state becomes glassy. 
Gillin and Sherrington [51] and Gillin et al. [52] have been able to analyze both the statics and the dynamics of several classes of mean-field spin glass models with a competition between glassy and ferromagnetic states (see also ref. [53] for a special case). Some features of the phase diagram of our model that we have been able to discover so far are also seen in their models.

Gillin et al. also studied full (as well as 1-step) replica symmetry breaking solutions, which we have not. In some of their models, the counterpart of our glassy native phase undergoes full RSB at low temperatures, and the counterpart of our II-VI boundary becomes vertical. It is possible in our model as well that, in particular regions of the phase diagram, our 1-step solutions are not stable and full RSB is necessary. More generally, it will be an interesting problem to try to explore what kinds and degrees of universality there are in the phase structures of various systems where the glassiness induced by disorder competes with some kind of order analogous to the native state in our problem or the ferromagnetic state in theirs.

\section{ACKNOWLEDGMENTS}

It is a pleasure to thank Silvio Franz for discussions leading to our formulation of this problem.

\section{APPENDIX A: CORRELATION FUNCTION $G_{10}^{S S^{\prime}}$}

Here we derive Eq. (30). Inserting Eq. (14) for the superfield into (25) gives

$$
\begin{aligned}
G_{10}^{s s^{\prime}}= & \left\langle x\left(s, t_{1}\right) x_{0}\left(s^{\prime}\right)\right\rangle+\left\langle\bar{\eta}\left(s, t_{1}\right) x_{0}\left(s^{\prime}\right)\right\rangle \theta_{1}+ \\
& +\bar{\theta}_{1}\left\langle\eta\left(s, t_{1}\right) x_{0}\left(s^{\prime}\right)\right\rangle+\bar{\theta}_{1} \theta_{1}\left\langle\tilde{x}\left(s, t_{1}\right) x_{0}\left(s^{\prime}\right)\right\rangle .
\end{aligned}
$$

One can show that the action of the dynamical generating functional $F_{d y n}$ (see Eq. 23) is invariant under the infinitesimal transformation (BRS symmetry)

$$
\delta \Phi(s, t, \theta, \bar{\theta})=\epsilon \frac{\partial}{\partial \bar{\theta}} \Phi(s, t, \theta, \bar{\theta}) .
$$

This follows in two steps. First one notices that for any function $f$

$$
\delta \int d \bar{\theta} f(\Phi(\bar{\theta}))=\epsilon \int d \bar{\theta} \frac{\partial}{\partial \bar{\theta}} f(\Phi(\bar{\theta}))=0,
$$

due to the identity $\int d \bar{\theta} \frac{\partial}{\partial \theta}=0$. This means that any term involving a local function of $\Phi$ (i.e., not containing derivatives over $\theta$ and $\bar{\theta}$ ), e.g., $S_{2}\left[\Phi, x_{0}\right]$ (see Eq. 16), is invariant under the transformation $(\mathrm{A} 2) . S_{0}\left[x_{0}\right]$ is trivially invariant since it does not contain the superfield
$\Phi$. (The same reasoning holds for a transformation like (A2) with a derivative with respect to $\theta$ instead of with respect to $\bar{\theta}$.) The only term left is the $S_{1}[\Phi]$ (i.e., the part of the action quadratic in the superfield) and it is straightforward to see that this term is also invariant under (A2) (though not under the transformation involving the derivative with respect to $\theta$ ).

The fact that the action is invariant under (A2) implies the Ward identity

$$
\frac{\partial}{\partial \bar{\theta}_{1}} G_{12}^{s s^{\prime}}=0
$$

which gives $0=\left\langle\eta x_{0}\right\rangle+\theta_{1}\left\langle\tilde{x} x_{0}\right\rangle$ (we have suppressed the arguments of the fields to simplify the notation). This implies that separately one has

$$
\left\langle\eta x_{0}\right\rangle=0, \quad\left\langle\tilde{x} x_{0}\right\rangle=0
$$

Inserting (A5) into (A1) gives the desired result, Eq. (30).

\section{APPENDIX B: DETAILS ON THE GVA AND HOW TO IMPROVE IT}

Here we give more background on the use of Eq. (36). In the dynamical calculation, the fields are complex and contain Grassmann variables; thus, $F_{d y n}$ is not a real number. This means that any interpretation of Eq. (36) as an extremum condition for $F_{d y n}$ has to be given up. Nevertheless, we can still make some sense of the GVA as the first step in a systematic approximation scheme.

Formally, one starts from Eq. (23), which we rewrite in the shorter form

$$
e^{-F_{d y n}}=\int D \Psi e^{-S[\Psi]} .
$$

where $\Psi$ stands for the pair $\left(x_{0}, \Phi\right)$ and, likewise, $D \Psi$ for $D x_{0} D \Phi$. One can express $F_{d y n}$ in a slightly different form

$$
e^{-F_{d y n}}=\left\langle e^{-\left(S-S_{v a r}\right)}\right\rangle_{v a r} e^{-F_{v a r}}
$$

where

$$
e^{-F_{v a r}}=\int D \Psi e^{-S_{v a r}}
$$

and

$$
\langle(\ldots)\rangle_{v a r}=\frac{\int D \Psi(\ldots) e^{-S_{v a r}}}{\int D \Psi e^{-S_{v a r}}}
$$

In a static calculation one would proceed with the inequality 


$$
e^{-F} \geq e^{-\left\langle\left(S-S_{v a r}\right)\right\rangle_{v a r}} e^{-F_{v a r}}
$$

to conclude that

$$
F \leq\left\langle S-S_{v a r}\right\rangle_{v a r}+F_{v a r}
$$

Thus, in a static calculation, the variationally-obtained $F$ gives an upper bound on the true $F$. What is allowed to vary is the form of $S_{v a r}$, most often, the parameters describing it. (In the GVA, $S_{v a r}$ is specified by $G_{12}^{s s^{\prime}}$ and $G_{10}^{s s^{\prime}}$.)

In the dynamical problem we follow another route, starting exactly at the problematic step, Eq. (B5), along the lines of ref. [54]. Instead of the inequality (B6) we use Eq. (B2) in a slightly modified form

$$
F_{d y n}=F_{v a r}-\ln \left\langle e^{-\Delta S}\right\rangle_{v a r},
$$

where $\Delta S=S-S_{v a r}$. Applying a cumulant expansion

$$
\begin{aligned}
\langle\exp (-\Delta S)\rangle_{\text {var }} & =\exp \left[-\langle\Delta S\rangle_{\text {var }}+\right. \\
& \left.+\frac{1}{2}\left(\left\langle\Delta S^{2}\right\rangle_{\text {var }}-\langle\Delta S\rangle_{\text {var }}^{2}\right)+\cdots\right],
\end{aligned}
$$

one gets

$$
F_{d y n}=F_{v a r}+\left\langle S-S_{v a r}\right\rangle_{v a r}+\Delta F
$$

where $\Delta F$ contains second and higher order corrections in $\Delta S$. In any approximation made by keeping a finite number of terms in (B8) (the simplest being to set $\Delta F=0), F_{d y n}$ depends on $G_{12}^{s s^{\prime}}$. To minimize this dependence, we chose $G_{12}^{s s^{\prime}}$ so that the derivative of the approximate form for $F_{d y n}$ with respect to $G_{12}^{s s^{\prime}}$ vanishes. This gives Eq. (36). Furthermore, if all terms in $\Delta F$ are kept, this procedure, by construction, formally gives back the exact $F_{d y n}$.

The meaning of minimizing the dependence with respect to quantities involving Grassmann numbers may seem obscure, but we note that we are using the SUSY representation only for compactness. The entire GVA calculation could have been presented equivalently without any Grassmann variables, with no change in meaning or result. Thus, we are really only minimizing the dependence on parameters of physically well-defined correlation and response functions.

[1] B. Rensberger, Life itself: exploring the realm of the living cell, (Oxford University Press, 1996).

[2] V.S. Pande, A.Y. Grosberg and T. Tanaka, Rev. Mod. Phys. 72, 259-314 (2000).
[3] P.G. Wolynes, H. Frauenfelder and R.H. Austin, More things in heaven and earth. A celebration of physics at the millennium, (Springer, 1999), page 706-725.

[4] P.G. Wolynes and W.A. Eaton, Physics World 9, 39 (1999).

[5] M. Karplus and E. Shakhnovich, in Protein Folding, edited by T.E. Creighton, ( Freeman, New York, 1992), page 127 .

[6] E. I. Shakhnovich and A. M. Gutin, Europhys. Lett. 8, 327 (1989).

[7] E. I. Shakhnovich and A. M. Gutin, J. Phys. A 22, 1647 (1989).

[8] T. Garel, D.A. Huse, L. Leibler, H. Orland, Europhys. Lett. 6, 307 (1988).

[9] T. Garel, H. Orland and E. Pitard, cond-mat/9706125.

[10] T. Garel, L. Leibler and H. Orland, J. Phys. II (France) 4, 2139 (1994).

[11] S. Takada and P.G. Wolynes, Phys. Rev. E 55, 4562 (1997).

[12] C.D. Sfatos, A.M. Gutin and E.I. Shakhnovich, Phys. Rev. E 48, 465-475 (1993).

[13] M. Sasai and P.G. Wolynes, Phys. Rev. Lett. 65, 2740 (1990).

[14] N. Lee and D. Thirumalai, J. Chem. Phys. 113, 51265129 (2000).

[15] E. Pitard, Eur. Phys. J B 7, 665-673 (1999).

[16] S. Takada, J.J. Portman and P.G. Wolynes, Proc. Natl. Acad. Sci. USA 94, 2318 (1997).

[17] D. Thirumalai, V. Ashwin and J.K. Bhattacharjee, Phys. Rev. Lett. 77, 5385-5388 (1996).

[18] E. Pitard and E.I. Shakhnovich, Phys. Rev. E 63, 041501 (2001).

[19] A.I.Olemskoi, Physica A 270, 444-452 (1999).

[20] A.I.Olemskoi, V.A.Brazhnyi, Physics of the Solid State 43, 386-396 (2001).

[21] M. Mezard, G. Parisi and M.A. Virasoro, Spin Glass Theory and Beyond (World Scientific, 1987).

[22] Z. Konkoli, J. Hertz and S. Franz, Phys. Rev. E 64, 051910 (2001).

[23] J.D. Bryngelson and P.G. Wolynes, Proc. Natl. Acad. Sci. USA 84, 7524 (1987).

[24] S. Ramanathan and E. Shakhnovich, Phys Rev E 50A, 1303-1312 (1994).

[25] V.S. Pande, A.Y. Grosberg and T. Tanaka, J. Phys. II 4, 1771-1784 (1994).

[26] J. Wilder and E.I. Shakhnovich, Phys. Rev. E 62B, 71007110 (2000).

[27] P.C. Martin, E.D. Siggia and H.A. Rose, Phys. Rev. A 8, 423 (1973).

[28] C. De Dominicis, Phys. Rev. B 18, 4913 (1978).

[29] H. K. Janssen, On the Renormalized Field Theory of Nonlinear Critical Relaxation, in From Phase Transitions to Chaos, page 68-91, year 1992, eds. Györgyi and I. Kondor and L. Sasvári and T. Tel, World Scientific, Singapore

[30] H. K. Janssen, Field-theoretic method applied to critical dynamics, in Dynamical Critical Phenomena and Related Topics, page 25-47, year 1979, ed. C. P. Enz, SpringerVerlag, Berlin

[31] J. Kurchan, J. Phys. I (France) 2, 1333 (1992). 
[32] M. Mézard and G. Parisi, J. Phys. I 1, 809 (1991).

[33] M. Mézard and G. Parisi, J. Phys. I 2, 2231 (1992).

[34] L.F. Cugliandolo, J. Kurchan and P. Le Doussal, Phys. Rev. Lett. 76, 2390 (1996).

[35] L.F. Cugliandolo and P. Le Doussal, Phys. Rev. E 53, 1525 (1996).

[36] N. Go, Biopolymers 20, 991-1011 (1981).

[37] Y. Ueda, H. Taketomi and N. Go, Int. J. Peptide. Res. 7,445 (1975).

[38] A.I.Olemskoi, V.A.Brazhnyi, Physica A 273, 368-400 (1999).

[39] L.F. Cugliandolo and J. Kurchan, Phys. Rev. Lett. 71, 173 (1993), J. Phys. A 27, 5749 (1994).

[40] A. Baldassarri, L.F. Cugliandolo, J. Kurchan and G. Parisi, J. Phys. A 27, 5749 (1994).

[41] L.F. Cugliandolo and J. Kurchan, Phil. Mag. B 71, 501 (1995).

[42] A. Crisanti and H.-J. Sommers, Z. Phys. B 87, 341 (1992).

[43] A. Crisanti, H. Horner and H.-J. Sommers, Z. Phys. B 92, 257 (1993).

[44] J.D. Bryngelson and P.G. Wolynes, Biopolymers 30, 177 (1990).

[45] S. Takada and P.G. Wolynes, J. Chem. Phys. 107, 9585 (1997).

[46] J.J. Portman, S. Takada and P.G. Wolynes, J. Chem. Phys. 114, 5069, 5082 (2001).

[47] R.A. Goldstein, Z.A. Luthey-Schulten and P.G. Wolynes, Proc. Nat. Acad. Sci. USA 89, 4918 (1992).

[48] J.D. Bryngelson and P.G. Wolynes, Proc. Nat Acad. Sci. USA 84, 7524 (1987).

[49] I.E.T. Iben, D. Braunstein, W. Doster, H. Frauenfelder, M.K. Hong, J.B. Johnson, S. Luck, P. Ormos, A. Schulte, P.J. Steinbach, A.H. Xie, and R.D. Young, Phys. Rev. Lett. 62, 1916 (1989).

[50] J.R.L. de Almeida and D.J. Thouless, J. Phys. A 11, 983 (1978).

[51] P. Gillin and D. Sherrington, J. Phys A 33, 3081 (2000).

[52] P. Gillin, H. Nishimori and D. Sherrington, J. Phys. A 34, 2949 (2001).

[53] J.A. Hertz, D. Sherrington and Th.M. Nieuwenhuizen, Phys. Rev. E 60, 2460 (1998).

[54] H. Kleinert, Path integrals in quantum mechanics, statistics and polymer physics, (World Scientific, 1995). 


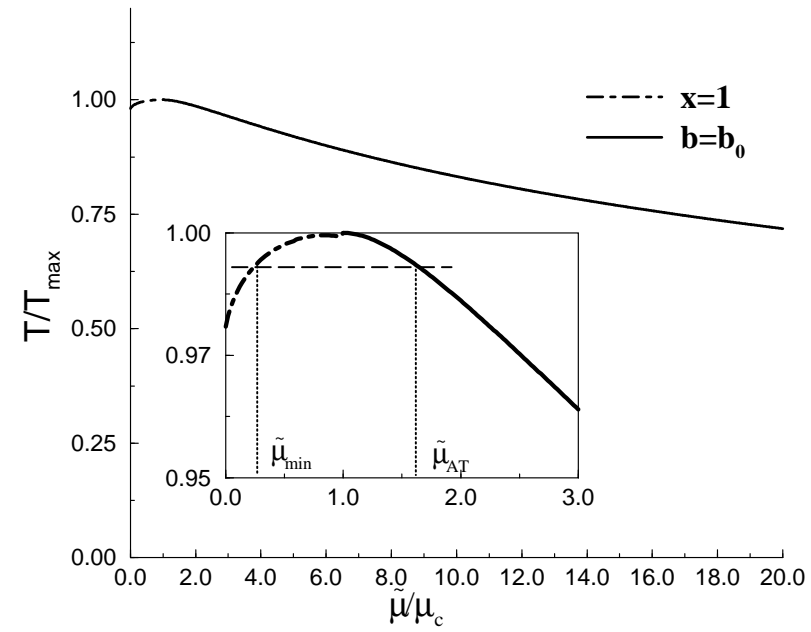

FIG. 1. Boundary of the glassy phase in the $(\tilde{\mu}, T)$ plane. The boundary is same as in the case of the random-heteropolymer model from paper I, except that native-state correlations lead to the replacement of $\mu$ by $\tilde{\mu}$. We have used parameters $d=3$ and $\sigma=1$. $T_{\max }$ is the maximum $T$ for which Eq. (80) has a solution (see Paper I for further details). $\mu_{c}$ is the value of $\tilde{\mu}$ where $T(\tilde{\mu})$ attains this maximum. The solid part of the boundary is an AT line, and the dash-dotted part marks a transition where $x \rightarrow 1$. Approaching the AT line from below, $b-b_{0} \rightarrow 0$, while $x$ remains strictly less than 1 . Approaching the $x=1$ line from below, $x \rightarrow 1$ smoothly, while $b-b_{0}$ is discontinuous there. Above both lines, $b=b_{0}$ and $x$ is undetermined (any $x \neq 0$ solves (74)-(80), and no physical quantity depends on it). The same holds for all figures where these lines appear.
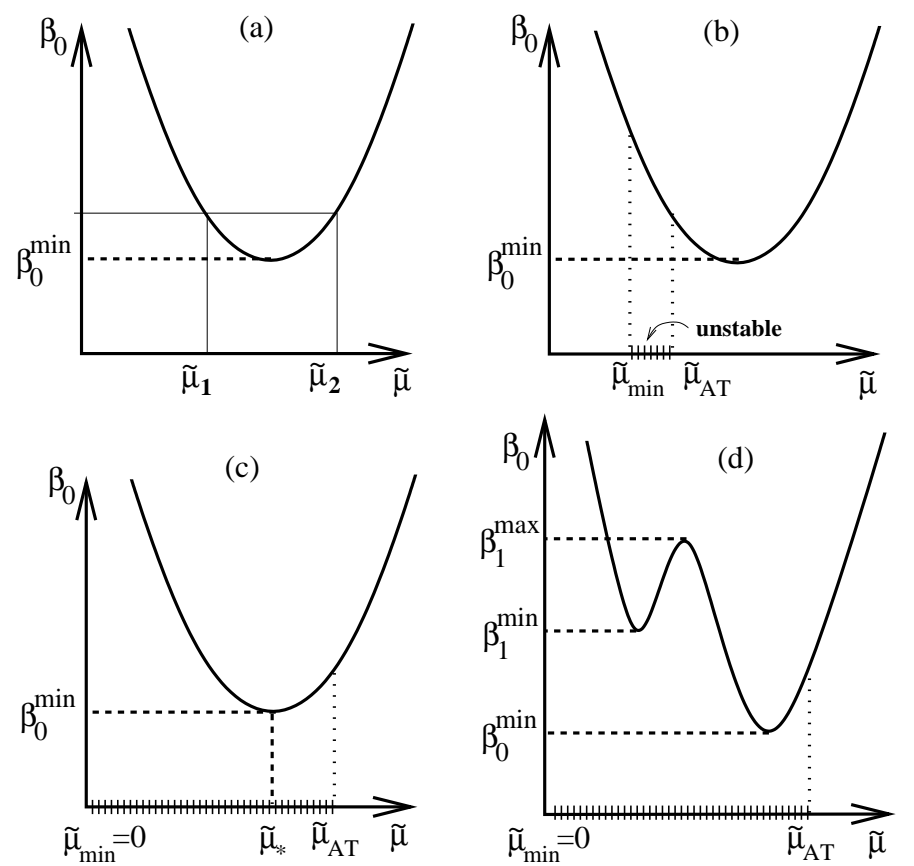

FIG. 2. Analysis of ergodic-native solutions: $\beta_{0}(\tilde{\mu}, T)$ as function of $\tilde{\mu}$ for four values of $T$ (see text for explanation). Panel (d) shows the existence of the two extra solutions (one stable, the other unstable) in the range $\left[\beta_{1}^{\min }, \beta_{1}^{\max }\right]$.
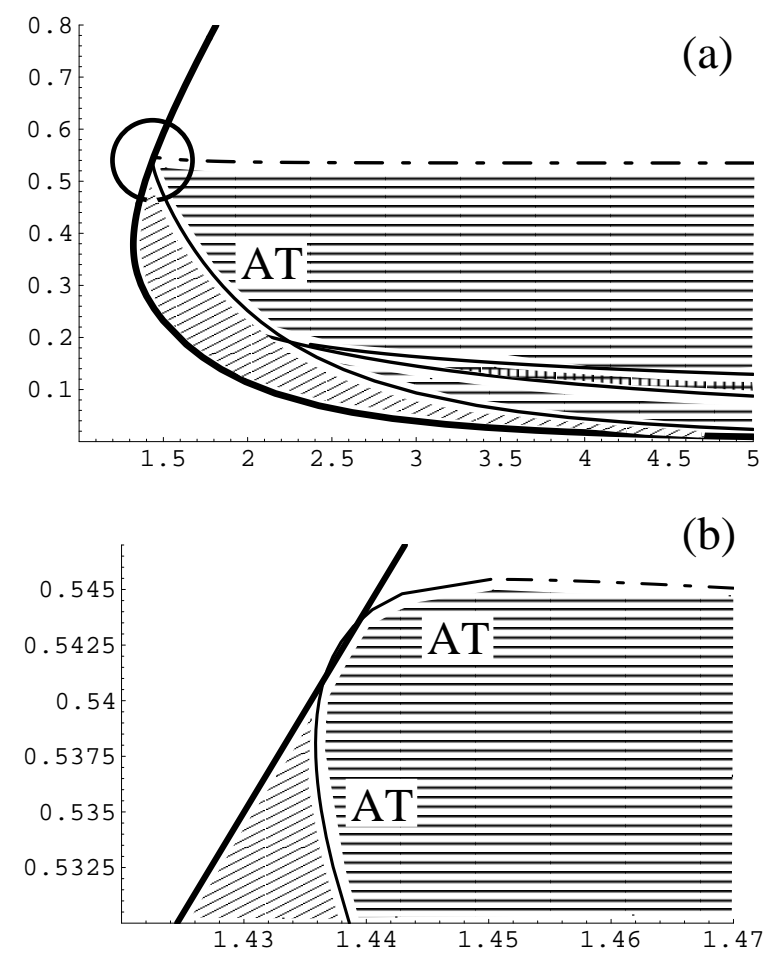

FIG. 3. Regions of existence and stability of ergodic-native solutions. Panel (a): Ergodic-native-phase and US solutions exist everywhere to the right of the thick solid curve. The ergodic native phase is stable against glassiness everywhere there except in the diagonally cross-hatched region. The US states are also unstable against glassiness there, and additionally in the horizontally cross-hatched region. The vertical cross-hatching marks the region where the extra phase seen in Panel (d) of Fig. 2 is found. (This phase is never stable against glassiness.) Panel (b): Enlargement of the circled region in Panel (a). The AT line is tangent to the ergodic phase boundary (thick line). At its maximum, at $T_{\max }$, it becomes an $x=1$ line (dashed-dotted line, see also Fig. 1). Lowering $T$ from the white region into the horizontally cross-hatched region results in two different types of transitions depending on whether one crosses the AT or the $x=1$ line. In both cases the US state becomes glassy. 

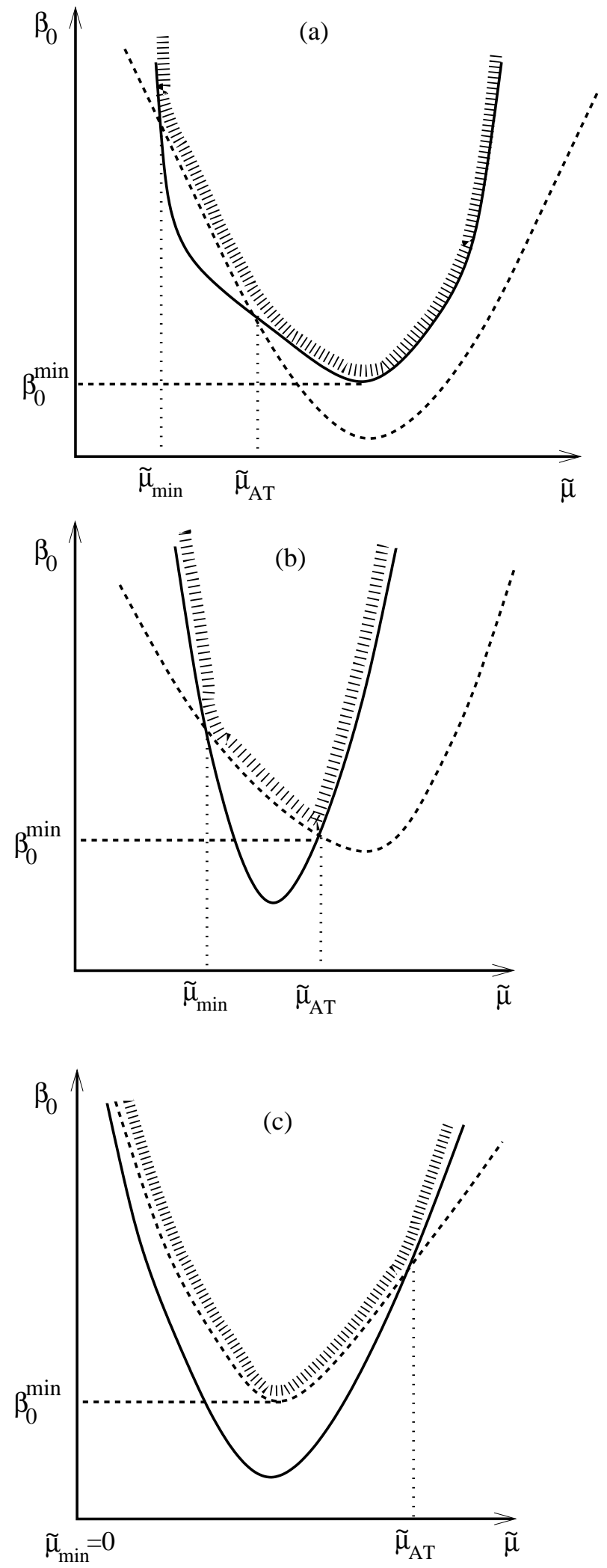

FIG. 4. Analysis of glassy-native solutions: $\beta_{0}(\tilde{\mu}, T)$ as a function of $\tilde{\mu}$ for three fixed values of $T$ (see text for explanation). Full lines: $\beta_{0}(\tilde{\mu}, T)$ calculated within the ergodic ansatz (as in Fig. 2). Dashed lines: $\beta_{0}(\tilde{\mu}, T)$ calculated with the glassy ansatz. The actual curves vary with $\tilde{\mu}$ in a way that is difficult to plot in a useful way, so here we have distorted them in such a way as to make their qualitative form (number and ordering of maxima and minima) evident. When the two curves cross at $\tilde{\mu}=\tilde{\mu}_{A T}$, one has to change from the ergodic to the glassy solution (when approaching from $\tilde{\mu}=\infty$ ). Similarly, when $\tilde{\mu}=\tilde{\mu}_{\text {min }}$ one has to go back to the ergodic solution. The thick dashed line indicates the physically relevant states (both stable phases and US states).
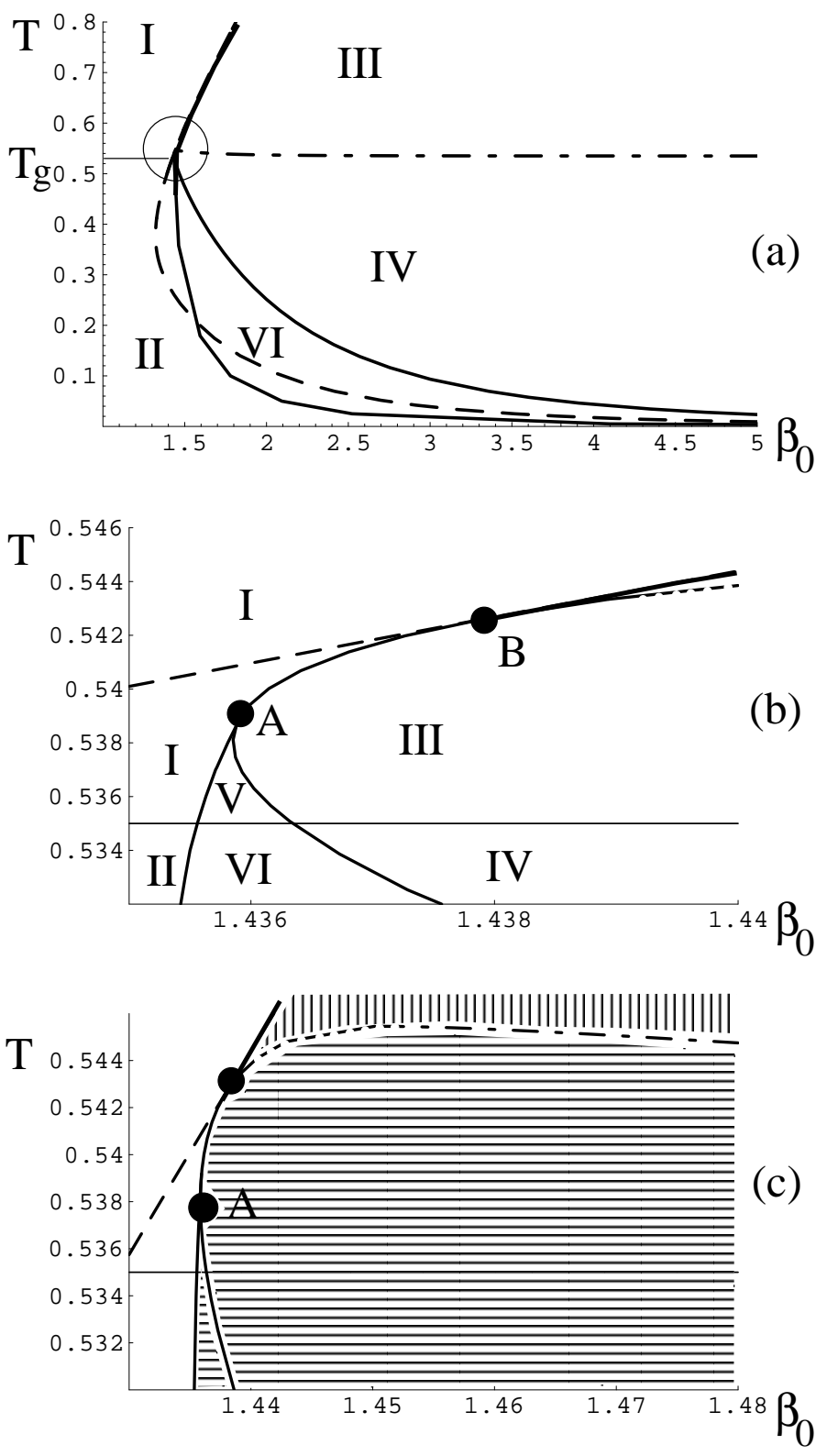
FIG. 5. The final phase diagram. Panel (a): Stable phases. Region I: Random globule is the only stable phase. Region II: Frozen globule is the only stable phase. Region III: Ergodic native and random globule phases stable. Region IV: Ergodic native and frozen globule phases stable. Region V (only visible in panel (b)): Glassy native and random globule stable. Region VI: Glassy native and frozen globule stable. The dashed line marks the boundary of the (unphysical) ergodic native state from Fig. 3, to emphasize that the phase boundary of the glassy native state (solid) does not coincide with it. Panel (b): Enlargement showing structure in the region near $T=T_{\max } \approx T_{g}$ and $\beta_{0}=1.45$ (including region $\mathrm{V})$. Below point $\mathrm{B}$ the boundary of region III is given by the AT line. Above point B the boundary is the ergodic-native stability limit (the uppermost line in Panel (a)). The continuation of the AT lie is shown as a dotted line (which turns into dash-dot $x=1$ line). Panel (c): US states are ergodic in the vertically hatched region, glassy in the horizontally hatched region. The boundary above and to the right of point $\mathrm{A}$ is an AT line. Beyond the region shown the $x=1$ line falls off monotonically, and for $\beta_{0} \rightarrow \infty$ it approaches $T_{g}$. Below point $\mathrm{A}$, the small- $\beta_{0}$ boundary coincides with the line between regions II and VI in panels (a) and (b).
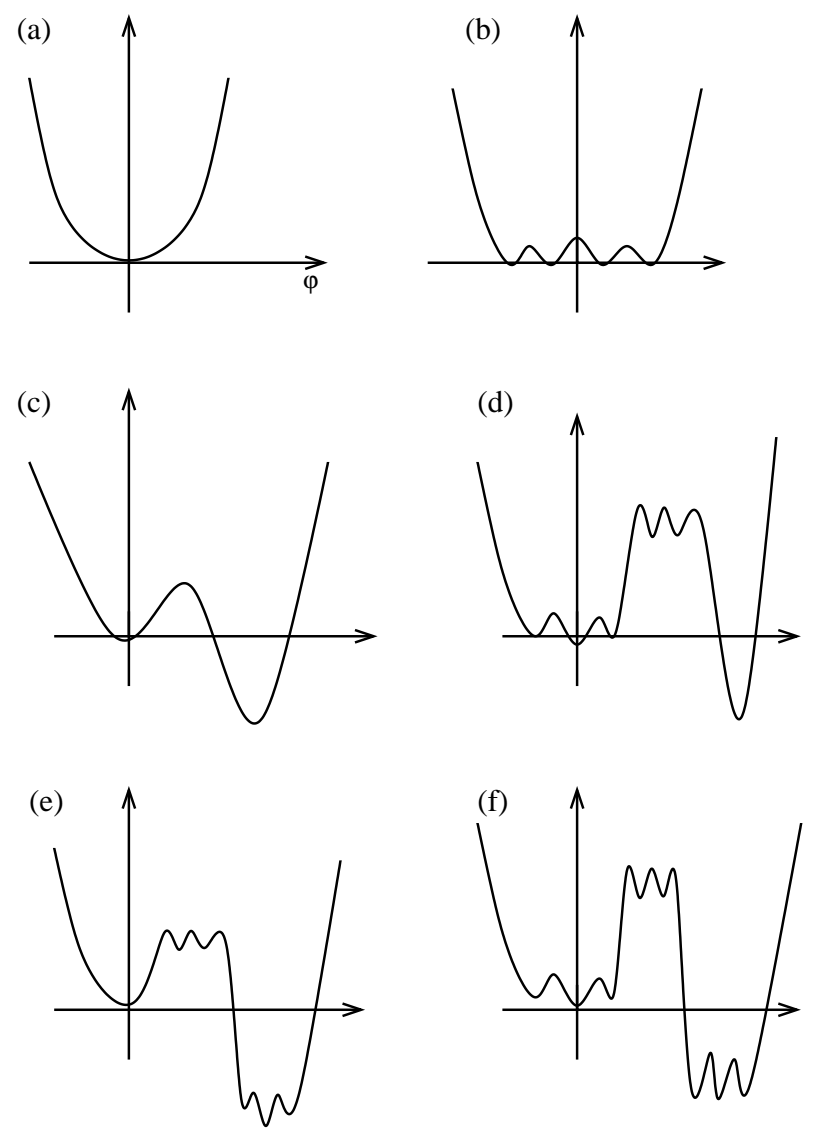

FIG. 6. Schematic free energy surfaces in different regions of the phase diagram. (See text for explanation.) (a): region I. (b): Region II. (c): Region III. (d): Region IV. (e): Region V. (f): Region VI. 